\title{
Molecular snapshots of drug release from tubulin over eleven orders of magnitude in time
}

Maximillian Wranik ${ }^{1}$, Tobias Weinert ${ }^{1}$, Chavdar Slavov ${ }^{2}$, Tiziana Masini ${ }^{3}$, Antonia Furrer ${ }^{1}$, Natacha Gaillard $^{1}$, Dario Gioia ${ }^{3}$, Marco Ferrarotti ${ }^{3}$, Daniel James ${ }^{1}$, Hannah Glover ${ }^{1}$, Melissa Carrillo ${ }^{1}$, Demet Kekilli $^{1}$, Robin Stipp ${ }^{1}$, Petr Skopintsev ${ }^{1}$, Steffen Brünle ${ }^{1}$, Tobias Mühlethaler ${ }^{1}$, John Beale ${ }^{1}$, Dardan Gashi ${ }^{4}$, Karol Nass ${ }^{4}$, Dmitry Ozerov 5 , Philip J.M. Johnson ${ }^{4}$, Claudio Cirelli ${ }^{4}$, Camila Bacellar ${ }^{4}$, Markus Braun ${ }^{2}$, Meitian Wang ${ }^{4}$, Florian Dworkowski ${ }^{4}$, Chris Milne, ${ }^{4}$ Andrea Cavalli3 ${ }^{3,6}$, Josef Wachtveitl ${ }^{2}$, Michel O. Steinmetz ${ }^{1,7, *}$ Jörg Standfuss ${ }^{1, *}$

${ }^{1}$ Division of Biology and Chemistry, Paul Scherrer Institut, 5232 Villigen, Switzerland.

${ }^{2}$ Institute of Physical and Theoretical Chemistry, Goethe University, Frankfurt am Main, Germany.

${ }^{3}$ Computational \& Chemical Biology, Istituto Italiano di Tecnologia, 16163 Genova, Italy.

${ }^{4}$ Photon Science Division, Paul Scherrer Institut, 5232 Villigen, Switzerland.

${ }^{5}$ Scientific Computing, Theory and Data, Paul Scherrer Institut, 5232 Villigen, Switzerland.

${ }^{6}$ Department of Pharmacy and Biotechnology, University of Bologna, 40126 Bologna, Italy.

${ }^{7}$ Biozentrum, University of Basel, 4056 Basel, Switzerland.

*Correspondence to: michel.steinmetz@psi.ch and joerg.standfuss@psi.ch

\section{Abstract}

The dynamic interplay between proteins and their ligands is central to molecular biology, pharmacology, and drug development but is difficult to resolve experimentally. Using timeresolved serial crystallography at a synchrotron and X-ray laser, we studied the release of the photochemical affinity switch azo-Combretastatin A4 from the anti-cancer target tubulin. Thirteen logarithmically spaced temporal snapshots at near-atomic resolution are complemented by timeresolved spectroscopy and molecular dynamics simulations. They show how the photoinduced cis to trans isomerization of the azobenzene bond stretches the ligand in the picosecond to nanosecond range, followed by stepwise opening of a gating loop within microseconds, and completion of the unbinding reaction within milliseconds. Ligand unbinding is accompanied by collapse of the binding pocket and global tubulin-backbone rearrangements. Our results have implications for the molecular basis of photopharmacology, the mechanism of action of anti-tubulin drugs and provide a general experimental framework to study protein-ligand interaction dynamics.

\section{One-Sentence Summary}

35 Time-resolved crystallography reveals structural changes in tubulin upon release of a photoswitchable microtubule inhibitor. 


\section{Main Text}

The dynamic interaction of small molecule ligands with their target proteins is the basis for numerous physiological processes in living cells. To catalyze chemical reactions, enzymes have to bind their substrates and release the products. Cellular signaling is coordinated by the binding of ligands that activate their cognate receptors in the cytoplasm or the cellular membrane. Small molecule ligands that act as drugs to selectively intercede in these physiological processes provide the most common strategy for medical intervention.

Much effort has been spent on resolving the molecular structure of important protein targets in complex with their ligands, yet it has been more challenging to experimentally follow the structural dynamics of protein-ligand binding and release with atomic details. Traditional structural biology relies on X-ray crystallography, nuclear magnetic resonance, or cryo-electron microscopy to provide accurate structures that can act as starting points for molecular dynamics (MD) simulations to study how a molecular system evolves with time. However, computational methods are limited in the size and time ranges that can be studied and can lead to results that are difficult to verify experimentally. Indirect verification can be achieved by mutagenesis followed by functional analyses (1) or a variety of spectroscopic methods that are commonly used to study protein-ligand interaction dynamics (2). Some of these methods allow for high temporal resolution, yet do not allow obtaining the direct atomic insight obtainable by structural biology.

Time-resolved serial crystallography combines the advantages of structural biology and spectroscopy to provide molecular snapshots over time that can be assembled into flipbook-like movies of how proteins function. Reactions from femtoseconds onwards have been probed at Xray free electron lasers (XFELs) and adaptations to the use at synchrotron sources have been very successful in reaching longer temporal regimes (3). In its most common form, time-resolved crystallography relies on an optical pump laser to initiate the reaction followed by a probing X-ray pulse to obtain structural information at a precise time delay after photoactivation. For this reason, the method has been most successful when studying photoactive proteins which are easy to trigger but are rare in biology (4). Besides these naturally evolved, photoactive protein systems, progress in chemical research has provided a large repertoire of synthetic photoswitches, whose structures are altered by the absorption of a photon. When incorporated into different types of polymers, such photochemical switches allow for exciting new applications (5). In chemistry, they can be used to control solid-to-liquid transitions ( 6 ). Biologists in turn have adapted synthetic photoswitches for the optical control of peptide (7) and protein functions (8) up to the neuromodulation of living 
mice (9). Finally, the field of photopharmacology uses photochemical affinity switches to localize therapeutic drug dose and reduce harmful side-effects (10).

A prominent example is the photochemical affinity switch azo-combretastatin (azo-CA4), which binds the $\alpha \beta$-tubulin heterodimer (hereafter called tubulin) (11). Tubulin is the key building block of the microtubule cytoskeleton in all eukaryotic cells. It is critical for cell division and thus a prominent target for cytotoxic drugs including the first line anti-cancer drug Paclitaxel (Taxol ${ }^{\circledR}$ ). Combretastatin A4 phosphate is strong cell growth inhibitor (12) that has been tested in clinical trials against different types of tumors (http://clinicaltrials.gov). It expresses anti-cancer activity via a destabilizing effect on microtubules through binding to the colchicine site located at the interface between the $\alpha$ and $\beta$ subunits of tubulin $(13,14)$. Azo-CA4 is a combretastatin A4 variant, in which the central $\mathrm{C}=\mathrm{C}$ stilbene bond is replaced by a more photoreactive $\mathrm{N}=\mathrm{N}$ azobenzene bond, allowing for reversible switching between a bent cis and straight trans form (11). Isomerization of an azobenzene bond occurs with high quantum efficiency and results in pronounced changes in its geometry and dipole moment within a few hundred femtoseconds after photons have been absorbed (15). Once built into azo-CA4, this effect allows to perturb the division of cancer cells using a localized pulse of monochromatic light (11). However, we have only just begun to understand how azobenzene isomerization changes binding affinity and how this then affects the conformational plasticity needed during microtubule formation.

Here, we describe how we used time-resolved serial crystallography at the Swiss Light Source (SLS) and the Swiss X-ray Free Electron Laser (SwissFEL) to follow the photoflash induced release of azo-CA4 from the colchicine binding site of tubulin with near-atomic spatial and subpicosecond temporal resolution. We resolved the evolution of the unbinding reaction in thirteen structural snapshots ranging from 1 ps to $100 \mathrm{~ms}$ after photoconversion of the compound. Our results reveal in atomic detail how a ligand is switched from high affinity to low affinity conformation and suggest the release mechanism. Molecular snapshots of the tubulin structural plasticity during ligand release may assist in finding the best strategy to target selective tubulin functions.

\section{Evolution of electron density features over time}

The X-ray crystallographic pump-probe data were collected on azo-CA4 soaked $\alpha \beta$-tubulinDARPin crystals $(16,17)$ at SwissFEL (18). Overall, we collected 1'330'681 indexed diffraction patterns with a resolution up to $1.7 \AA$ (Table S1). The data are divided into "dark" images collected 
without light exposure and images taken at time delays of 1 ps, 25 ps, 35 ps, 125 ps, $1 \mathrm{~ns}, 10 \mathrm{~ns}$, $100 \mathrm{~ns}, 1 \mu \mathrm{s}, 10 \mu \mathrm{s}, 100 \mu \mathrm{s}, 1 \mathrm{~ms}$, and $10 \mathrm{~ms}$ after illumination with a femtosecond flash of laser light (Fig. 1). In addition, we collected serial synchrotron crystallography data (19) on the ligandbound and ligand-free state obtained by approximately $100 \mathrm{~ms}$ of light exposure.

The difference electron density maps $\left(\mathrm{F}_{\text {obs }}(\right.$ light $)-\mathrm{F}_{\text {obs }}($ dark $\left.)\right)$ calculated from the data are of high quality (Fig. 2A, Movie S1) and can be quantitatively followed by Pearson correlation analysis to highlight transitions between structural intermediates (20) (Fig. S1). We observed four major blocks of elevated correlation in the picosecond, nanosecond, microsecond, and early millisecond time regimes. Further substructures can be derived when comparing changes in the binding pocket to backbone movements occurring over the whole protein. From this analysis, it is clear that illumination generates a nonequilibrium photoproduct population that relaxes over a broad time range from picoseconds to milliseconds.

Using structural refinements against extrapolated data from the light-activated fraction of the structure factors $(21,22)$, we were able to generate atomic models for predominant intermediate states (Fig. S2, Table S2). In addition, we used conventional refinement approaches to solve room temperature crystal structures of the initial cis-azo-CA4 isomer bound and ligand-free states. Taken together, our data provide a comprehensive overview of the structural changes occurring upon cis-trans isomerization and the following release of the azo-CA4 compound from its tubulinbinding pocket. A movie covering eleven orders of magnitude in time (Movie S2) summarizes the critical molecular rearrangements that we will discuss in the following sections.

\section{Early rearrangements in the binding pocket}

The conformation of a ligand is generally presumed to precisely fit the architecture of its binding pocket even though both protein and ligand are in constant motion. The photoinduced release of azo-CA4 provides an excellent system to study what happens when a ligand deviates from its primary binding pose. Before light-activation, the cis isomer of azo-CA4 is bound to the colchicine site at the interface between the $\alpha$ and $\beta$ subunits of tubulin (Fig. 1B). Interactions with the protein are primarily hydrophobic in nature, except for hydrogen bonds to the backbone carbonyl of $\alpha$ Thr179 and the backbone amide of $\alpha$ Val181, respectively (Fig. 2B). Further interactions are mediated by a water molecule interacting directly with the azobenzene bond of azo-CA4. Overall, the binding pose of the ligand is nearly identical to that of the parent compound combretastatin A4 that contains a stilbene instead of the azobenzene group connecting the A and B rings of the ligand 
(23). Difference electron density maps and structural refinements show that azo-CA4 has completed cis-trans isomerization already 1 ps after photoactivation (Fig. 2F and Movie S1). This reaction stretches the molecule by $1.9 \AA$ as measured by the increase in distance between the 4 and 4' atoms of its A and B rings, respectively (Fig. 2 C). Stretching changes the interaction pattern of the ligand with the protein as the binding pocket has not yet adapted to the altered ligand conformation. The water-mediated interactions are broken and relocation of the two ring moieties of azo-CA4 reduces their hydrophobic stacking interactions with the protein. From 25 ps onwards, we observed conformational rearrangements of the $\alpha$ Val181 side chain located close to ring B of azo-CA4, resulting in a rotamer change of this residue at $125 \mathrm{ps,} \mathrm{providing} \mathrm{additional} \mathrm{space} \mathrm{for}$ accommodating the stretched ligand in the binding pocket (Fig. 2D). From 1 ns onwards, the electron density maps suggest a larger conformational flexibility of azo-CA4 that propagates into a new relaxed ligand-binding pose observed at 100 ns (Fig. 1E). At this time point, ring A of azoCA4 is relocated by $2.6 \AA$ towards the $\beta$ T7 loop of $\beta$-tubulin, known to be involved in ligand unbinding $(17,23)$.

To further characterize azo-CA4 isomerization in context of the colchicine site, we have used timeresolved, transient absorption spectroscopy (Fig. 2F). In agreement with the structural results, the cis-trans isomerization proceeds with a lifetime of about $450 \mathrm{fs}$; a similar scale as observed for the same reaction in unsubstituted azobenzenes (15). At the $1 \mathrm{ps}$ delay, we have refined the crystal structure of the tubulin-azo-CA4 complex in the predominant state but the electron density indicates that azo-CA4 has not yet reached a single conformation. With the experimental framework described here and recent progress in using machine learning to analyze ultrafast XFEL data (24), it seems now within reach to study the relevance of this heterogeneity to the isomerization mechanism with temporal resolutions of only few femtoseconds.

Interestingly, the transient spectra of azo-CA4 taken in the presence of tubulin exhibit an additional pronounced blue shift with a lifetime of 35 ps (Fig. S3), indicating that the binding pocket further influences the ligand in this temporal regime. As the spectral changes coincide in time with the changed interaction patterns after cis-trans isomerization and the rotation of the $\alpha$ Val181 side chain, it seems likely that the ligand cannot stretch as quickly and needs longer to achieve a relaxed conformation within the confined space of the binding pocket. To bridge the gap between structural effects and binding energy we relied on MD simulations (25). We aimed at estimating the relative binding free energy difference between the cis and trans isomers $\left(\Delta \Delta \mathrm{G}_{\text {cis-trans }}\right)$ using the computational protocol utilized for the tubulin-combretastatin A4 
complex (23). Fig. 2G shows the free energy profiles corresponding to the bound and unbound isomerization processes of the thermodynamic cycle (cis-bound $\rightarrow$ trans-bound $\rightarrow$ trans-unbound $\rightarrow$ cis-unbound). As expected, the trans isomer is the most stable form in both the bound and unbound simulations with $\Delta \mathrm{G}$ values of $\Delta \mathrm{G}_{\text {cis-trans-bound }}=-14 \mathrm{kcal} / \mathrm{mol}$ and $\Delta \mathrm{G}_{\text {cis-trans-unbound }}=-19$ $\mathrm{kcal} / \mathrm{mol}$, respectively. The resulting relative binding free energy difference of $\Delta \Delta \mathrm{G}_{\text {cis-trans }}=5$ $\mathrm{kcal} / \mathrm{mol}$ clearly favors the cis-isomer stabilized interactions with tubulin. Such a free energy difference translates into an increased affinity of $\sim 4300$ times based on the equilibrium probability ratio and Boltzmann statistics. The low affinity of trans-CA4 explains why we were unable to crystallize this isomer in complex with tubulin using classical crystallography methods but instead needed a time-resolved experiment.

\section{Binding pocket rearrangements upon ligand release}

Both the induced fit and conformational selection models of ligand binding predict complex dynamic transitions associated with the ligand entering and leaving the binding pocket of its target protein $(26,27)$. The colchicine site of tubulin is no exception, as it displays pronounced conformational adaptations between the ligand-bound and -unbound forms of the protein $(14,23$, $28,29)$.

Our time-resolved structures allow to follow the reorganization of the colchicine site following formation of the metastable azo-CA4 binding pose. The relevant temporal regime starts at $100 \mathrm{ns,}$ when the A ring of the ligand has been repositioned into close contacts with residue $\beta$ Ala248 of the $\beta$ T7 loop (Fig. 3B). Initial conformational changes of the $\beta$ T7 loop follow on the microsecond scale with further rearrangements of the loop, including a displacement of $\beta$ Asn 247 and $\beta$ Leu 246 occurring in the millisecond time range (Fig. 3C,D). The binding pocket initially expands starting from a volume of $446 \AA^{3}$ before illumination (cis-azoCA4) to $552 \AA^{3}$ at $100 \mathrm{~ns}$ (relaxed transazoCA4), and then collapses from $363 \AA^{3}$ at $1 \mathrm{~ms}$ (after $\beta$ T7 loop movement) down to $71 \AA^{3}$ at 10 ms when the compound has left the binding pocket (Fig. 3G). Based on visual inspection of difference maps and plotting electron density changes on the ligand over time, we have modeled the release of azo-CA4 between the 1 and $10 \mathrm{~ms}$ time delays (Fig. 3D,E). However, residual ligand can still be observed at low occupancy in the $10 \mathrm{~ms}$ data and is only fully released at about $100 \mathrm{~ms}$

(Fig. 3F) as evident from the synchrotron structure which is essentially identical to the one determined in the absence of azo-CA4 (Fig. S4). The long release time is consistent with a 
stochastic nature of the process where unbinding events of individual molecules occur over several milliseconds in time once they are initiated on faster time scales.

The binding pocket is not freely accessible to the solvent but our structural analysis suggests the opening of an exit channel between $100 \mu$ s and 1 ms (Fig. 3H). Reorganization of the $\beta T 7$ loop, particularly through a rotation of the $\beta$ Leu246 side chain, results into a $8 \AA$ long channel from a possible exit site close to the A ring of the ligand (Fig. S5). The diameter of the channel is 4-6 A, which seems small given the $7 \AA$ diameter for the larger A ring of azo-CA4. As the unbinding takes place over many milliseconds throughout the crystal, it seems likely that the channel transiently widens in short-lived intermediates that did not sufficiently accumulate to be observed. To study this possibility, we have used MD simulations starting from the $1 \mathrm{~ms}$ delay where the opening of the channel is most prominent. Almost $50 \mu$ s of simulations suggests that the azo-CA4 approaches a position close to the proposed exit site of the protein, where only minimal changes would be sufficient to let the ligand diffuse out of its binding pocket. Once the ligand detaches our crystallographic data again shows how the $\beta$ T7 loop flips back and packs against $\beta$-tubulin residues that were previously engaged in azo-CA4 binding. Overall, our data are consistent with the flexible $\beta$ T7 loop to act as a "molecular gate" that opens and closes the colchicine site and, depending on its current conformation, directly competes with ligand binding $(14,29)$.

There are elements of an induced fit when the binding pocket adapts to the stretched trans azoCA4 within a microsecond. However, the collapse of the binding pocket coinciding with ligand release (Fig. 3G) suggests that the tubulin-azo-CA4 interaction dynamics are dominated by conformational selection involving open and closed positions of the $\beta T 7$ loop. A transiently formed conformation of the binding pocket is stabilized through the ligand, but relaxes when ligand-protein interactions are removed. Notably, all colchicine-site ligands structurally analyzed so far displace the $\beta$ T7 loop upon binding $(30,31)$, suggesting a general mechanism for this class of microtubule-destabilizing agents.

\section{Global tubulin backbone rearrangements}

Tubulin displays substantial structural plasticity that is vital to microtubule dynamics and thus its cellular function. A prominent example is the global conformational change during tubulin 30 assembly into microtubules from a "curved" free tubulin to a "straight" microtubule-lattice incorporated tubulin conformation (32). This major reorganization of the tubulin dimer is 
accompanied by a contraction of the colchicine site $(14,29)$. Binding of ligands including combretastatins to the colchicine site prevents its contraction and thus destabilizes microtubules by stabilizing the curved conformation of free tubulin (30).

To illustrate how such global backbone rearrangements develop with time, we superimposed the structures obtained from our thirteen time delays and visualized the rearrangements of $\mathrm{C} \alpha$ backbone atoms as individual vectors. We observed several motions that correspond well to our initial Pearson-correlation analysis of electron density changes, and which coincide in time with the major events in the ligand-binding pocket (Fig. S1). This result suggests that local structural changes in the binding pocket are accompanied by global, allosteric backbone rearrangements throughout the $\alpha$ - and $\beta$-tubulin subunits of the tubulin dimer.

Next, we assessed global conformational changes occurring between our tubulin structures obtained in the picosecond, nanosecond, microsecond, and millisecond time range (Fig. 4B). We found that global backbone changes in the picosecond time range, representing early adaptations following cis-trans isomerization of the ligand, are minor and do not go beyond the $>0.5 \AA$ displacements in $\mathrm{C} \alpha$ backbone atoms we introduced as a significance threshold for the vector analysis. However, global structural changes became more pronounced in the nanosecond time range, where we observed coordinated inward motions of both the $\alpha$ - and $\beta$-tubulin subunits. These changes correlated well to the time range where the A ring of azo-CA4 shifts to a new place completing the cis-to-trans relaxation process within the binding pocket. Interestingly, some of these movements are reverted in the microsecond time range, where the $\beta \mathrm{T} 7$ loop starts moving and finally collapses into the binding pocket. In the millisecond time range, final backbone movements primarily in the $\alpha$-tubulin subunit brings the structure back to its ligand-free state. To analyze whether the global backbone rearrangements in tubulin upon ligand release have an impact on the overall conformation of the protein, we analyzed the curvature of an oligomer constructed from three longitudinally, head-to-tail aligned tubulin dimers, as previously described (33) (Fig. 4B and Fig. S6). Like our initial structural comparison, this complementary analysis suggests no significant differences in the picosecond time regime while significant changes in curvature are observed in the nano- and millisecond time range. Once azo-CA4 is released the curvature is markedly reduced into the direction of the straight protofilament conformation found in 30 microtubules. Taken together, these analyses suggest that the global tubulin backbone movements, as well as overall changes in tubulin conformational curvature evolve in par with structural 
alterations of ligands within the colchicine binding site. This is in agreement with the inhibiting effect on microtubule formation exhibited by these small molecules.

\section{Conclusions}

Due to their broad biological relevance, structural biologists have determined thousands of atomicresolution structures of protein-ligand complexes. The pharmaceutical industry is relying on structural information as an integral part of a rational approach to discover new drugs and understand their mechanisms of action. Based on static structures alone, it is tempting to oversimplify the dynamic nature of ligand-binding and -unbinding reactions. The ligand residence time, for example, is considered one of the key factors for improving drug efficacy, as the lifetime of the binary drug-target complex, and not the binding affinity alone, dictates much of the in vivo pharmacological activity of a compound. Also, the probability of side effects can increase if a drug does not engage its biological target long enough $(34,35)$. Our molecular snapshots resolve the formation and dissolution of a metastable binding pose within the most frequently targeted drugbinding site in tubulin (30). They provide an excellent framework on which to explore the use of time-resolved structural information as a new dimension in structure-guided drug development.

Many new beamlines at $4^{\text {th }}$ generation synchrotron sources are purpose build for time-resolved serial crystallography and X-ray laser sources worldwide deliver more and more results (4). However, a major drawback has not been resolved: measurements typically rely on naturally light activated target proteins. While promising results using photo-caged compounds (36) and rapidmixing have been shown (37), these will not allow observations on very fast time scales relevant for protein-ligand interactions. Our study demonstrates that time-resolved crystallography is now efficient enough for an untargeted, logarithmic approach to cover many orders of magnitude in time on targets that have not been extensively characterized by spectroscopic methods. Key for the employed pump-probe approach is the use of photochemical affinity switches, for which azo-CA4 is just one example. We anticipate that similar compounds developed to control kinases, channels, G protein-coupled receptors, and other pharmaceutically relevant protein targets will be important future tools to experimentally study the structural plasticity of proteins, as well as protein-ligand interaction dynamics. 


\section{Acknowledgments}

We are grateful for the excellent support from the PSI Crystallization Facility and the Macromolecular Crystallography group during growing and testing of crystals at the Swiss Light Source. The Biomolecular Structure and Mechanism Program of the Life Science Zürich Graduate School is acknowledged for their academic framework for our graduate students.

\section{Funding}

This project was founded by the following agencies: The Swiss National Science Foundation project grants 31003A_179351 (to J.S.), to 310030_197674 (to T.W.), 310030_192566 (to M.O.S.) and the NCCR:MUST (to C.M. and J.S.), the Swiss Nanoscience Institute SNI \#1904 (to M.C.), Deutsche Forschungsgemeinschaft, Project WA 1850/4-3 (C.S. and J.W.).

\section{Author contributions}

The project was coordinated and led by J.S. following input on time-resolved serial crystallographic data acquisition and evaluation from T.W., computer simulations from A.C., and time-resolved spectroscopy from J.W. and tubulin structural biology from M.O.S.. The TD1 protein complex was purified and crystallized by M.W. with the help from A.F., N.G., and other members of the team during the large-scale preparation phase. The azo-CA4 compound was synthesized by T.M.. Crystal injection was optimized by M.W., A.F., and D.J.. The lipidic cubic phase injector was operated and aligned during the beamtime by A.F. D.G., and D.J.. The endstation including the laser system were aligned and operated by P.J.M.J., D.O., K.N., C.C., and C.M.. Data processing during the beamtime was initially done using the online data analysis pipeline established by K.N. and D.O. followed by manual optimization by S.B. and P.S. with instructions from T.W.. Final structures were refined by M.W. and T.W. and interpreted together with J.S.. Quantum chemical calculations were done by M.F. and D.G. under supervision of A.C.. The time-resolved spectroscopic experiments were done by N.G., T.W., and C.S. and interpreted together with M.B. and J.W.. The manuscript was written by J.S. with direct contributions from M.W., T.W., M.O.S., C.S., J.W., D.G., and A.C., and further suggestions from the other authors. All authors read and acknowledged the manuscript.

\section{Competing interests}

The authors declare that they have no competing interests. 


\section{Data and materials availability}

Coordinates and structure factors for tubulin in complex with cis-azo-CA4 have been deposited in the PDB database under accession code xxx. Light activated data sets, coordinates, light amplitudes, dark amplitudes and extrapolated structure factors have been deposited in the PDB database under accession codes $\mathrm{xxx}$ (1 ps), $\mathrm{xxx}(25 \mathrm{ps}), \mathrm{xxx}(35 \mathrm{ps}), \mathrm{xxx}(125 \mathrm{ps}), \mathrm{xxx}(1 \mathrm{~ns}), \mathrm{xxx}$ $(10 \mathrm{~ns}), \operatorname{xxxx}(100 \mathrm{~ns}), \operatorname{xxxx}(1 \mu \mathrm{s}), \operatorname{xxxx}(10 \mu \mathrm{s}), \operatorname{xxxx}(100 \mu \mathrm{s}), \operatorname{xxxx}(1 \mathrm{~ms})$ and $\operatorname{xxxx}(10 \mathrm{~ms})$. The serial synchrotron data have been deposited in the same format under $\mathrm{xxxx}$ (dark) and $\mathrm{xxxx}$ (100 ms).

\section{References}

1. S. A. Hollingsworth, R. O. Dror, Molecular Dynamics Simulation for All. Neuron 99, 1129-1143 (2018).

2. O. Bozovic et al., Real-time observation of ligand-induced allosteric transitions in a PDZ domain. Proc Natl Acad Sci U S A 117, 26031-26039 (2020).

3. G. Branden, R. Neutze, Advances and challenges in time-resolved macromolecular crystallography.

Science 373, 980-+ (2021).

4. A. M. Orville, Recent results in time resolved serial femtosecond crystallography at XFELs. Curr Opin Struc Biol 65, 193-208 (2020).

5. F. A. Jerca, V. V. Jerca, R. Hoogenboom, Advances and opportunities in the exciting world of azobenzenes. Nature Reviews Chemistry 6, 51-69 (2022).

6. H. Zhou et al., Photoswitching of glass transition temperatures of azobenzene-containing polymers induces reversible solid-to-liquid transitions. Nat Chem 9, 145-151 (2017).

7. S. Sporlein et al., Ultrafast spectroscopy reveals subnanosecond peptide conformational dynamics and validates molecular dynamics simulation. Proc Natl Acad Sci U S A 99, 7998-8002 (2002).

8. O. Bozovic, B. Jankovic, P. Hamm, Using azobenzene photocontrol to set proteins in motion. Nature

Reviews Chemistry 6, 112-124 (2022).

9. R. Durand-de Cuttoli et al., Manipulating midbrain dopamine neurons and reward-related behaviors with light-controllable nicotinic acetylcholine receptors. Elife 7, (2018).

10. K. Hull, J. Morstein, D. Trauner, In Vivo Photopharmacology. Chem Rev 118, 10710-10747 (2018).

11. M. Borowiak et al., Photoswitchable Inhibitors of Microtubule Dynamics Optically Control Mitosis and

12. G. R. Pettit et al., Isolation and structure of the strong cell growth and tubulin inhibitor combretastatin A-4. Experientia 45, 209-211 (1989).

13. G. M. Tozer, C. Kanthou, C. S. Parkins, S. A. Hill, The biology of the combretastatins as tumour vascular targeting agents. Int $J$ Exp Pathol 83, 21-38 (2002).

14. R. B. G. Ravelli et al., Insight into tubulin regulation from a complex with colchicine and a stathmin-like domain. Nature 428, 198-202 (2004).

15. T. Nagele, R. Hoche, W. Zinth, J. Wachtveitl, Femtosecond photoisomerization of cis-azobenzene. Chem Phys Lett 272, 489-495 (1997).

16. L. Pecqueur et al., A designed ankyrin repeat protein selected to bind to tubulin caps the microtubule plus

end. Proc Natl Acad Sci U S A 109, 12011-12016 (2012).

17. G. La Sala et al., Structure, Thermodynamics, and Kinetics of Plinabulin Binding to Two Tubulin Isotypes. Chem-Us 5, 2969-2986 (2019).

18. R. Abela et al., Perspective: Opportunities for ultrafast science at SwissFEL. Struct Dyn 4, 061602 (2017).

19. T. Weinert et al., Proton uptake mechanism in bacteriorhodopsin captured by serial synchrotron crystallography. Science 365, 61-65 (2019).

20. C. Wickstrand et al., A tool for visualizing protein motions in time-resolved crystallography. Struct Dyn 7, 024701 (2020).

21. S. Pandey et al., Time-resolved serial femtosecond crystallography at the European XFEL. Nature Methods 17, 73-+ (2020). 
22. P. Skopintsev et al., Femtosecond-to-millisecond structural changes in a light-driven sodium pump. Nature, (2020).

23. R. Gaspari, A. E. Prota, K. Bargsten, A. Cavalli, M. O. Steinmetz, Structural Basis of cis- and transCombretastatin Binding to Tubulin. Chem-Us 2, 102-113 (2017).

24. A. Hosseinizadeh et al., Few-fs resolution of a photoactive protein traversing a conical intersection. Nature 599, 697-701 (2021).

25. S. Decherchi, A. Cavalli, Thermodynamics and Kinetics of Drug-Target Binding by Molecular Simulation. Chem Rev 120, 12788-12833 (2020).

26. D. E. Koshland, Application of a Theory of Enzyme Specificity to Protein Synthesis. Proc Natl Acad Sci U

$S$ A 44, 98-104 (1958)

27. D. E. Koshland, Jr., G. Nemethy, D. Filmer, Comparison of experimental binding data and theoretical models in proteins containing subunits. Biochemistry 5, 365-385 (1966).

28. A. E. Prota et al., The Novel Microtubule-Destabilizing Drug BAL27862 Binds to the Colchicine Site of Tubulin with Distinct Effects on Microtubule Organization. Journal of Molecular Biology 426, 1848-1860 (2014).

29. A. Dorleans et al., Variations in the colchicine-binding domain provide insight into the structural switch of tubulin. P Natl Acad Sci USA 106, 13775-13779 (2009).

30. M. O. Steinmetz, A. E. Prota, Microtubule-Targeting Agents: Strategies To Hijack the Cytoskeleton. Trends Cell Biol 28, 776-792 (2018).

31. T. Muhlethaler et al., Comprehensive Analysis of Binding Sites in Tubulin. Angew Chem Int Ed Engl 60, 13331-13342 (2021).

32. M. Knossow, V. Campanacci, L. A. Khodja, B. Gigant, The Mechanism of Tubulin Assembly into Microtubules: Insights from Structural Studies. iScience 23, 101511 (2020).

33. G. J. Brouhard, L. M. Rice, The contribution of alphabeta-tubulin curvature to microtubule dynamics. $J$

Cell Biol 207, 323-334 (2014).

34. R. A. Copeland, The drug-target residence time model: a 10-year retrospective. Nat Rev Drug Discov 15, 87-95 (2016).

35. M. Bernetti, M. Masetti, W. Rocchia, A. Cavalli, Kinetics of Drug Binding and Residence Time. Annu Rev Phys Chem 70, 143-171 (2019).

36. P. Mehrabi et al., Time-resolved crystallography reveals allosteric communication aligned with molecular breathing. Science 365, 1167-1170 (2019).

37. S. Pandey et al., Observation of substrate diffusion and ligand binding in enzyme crystals using highrepetition-rate mix-and-inject serial crystallography. Iucrj 8, 878-895 (2021).

38. E. Nogales, K. H. Downing, L. A. Amos, J. Lowe, Tubulin and FtsZ form a distinct family of GTPases. Nat Struct Biol 5, 451-458 (1998).

39. E. H. Kellogg et al., Insights into the Distinct Mechanisms of Action of Taxane and Non-Taxane Microtubule Stabilizers from Cryo-EM Structures. J Mol Biol 429, 633-646 (2017).

40. M. Sugahara et al., Hydroxyethyl cellulose matrix applied to serial crystallography. Sci Rep 7, 703 (2017).

41. D. James et al., Improving High Viscosity Extrusion of Microcrystals for Time-resolved Serial Femtosecond Crystallography at X-ray Lasers. J Vis Exp, (2019).

42. K. Nass et al., Pink-beam serial femtosecond crystallography for accurate structure-factor determination at an X-ray free-electron laser. IUCrJ 8, (2021).

43. U. Weierstall et al., Lipidic cubic phase injector facilitates membrane protein serial femtosecond crystallography. Nat Commun 5, 3309 (2014).

44. O. B. Zeldin, M. Gerstel, E. F. Garman, RADDOSE-3D: time- and space-resolved modelling of dose in macromolecular crystallography. Journal of Applied Crystallography 46, 1225-1230 (2013).

45. T. A. White et al., CrystFEL: a software suite for snapshot serial crystallography. Journal of Applied Crystallography 45, 335-341 (2012).

46. T. A. White et al., Recent developments in CrystFEL. J Appl Crystallogr 49, 680-689 (2016).

47. Y. Gevorkov et al., XGANDALF - extended gradient descent algorithm for lattice finding. Acta Crystallogr A Found Adv 75, 694-704 (2019).

48. I. J. Tickle, Flensburg, C., Keller, P., Paciorek, W., Sharff, A., Vonrhein, C., Bricogne, G. . (Cambridge, United Kingdom: Global Phasing Ltd., http://staraniso.globalphasing.org/cgi-bin/staraniso.cgi, 2018).

49. P. D. Adams et al., PHENIX: building new software for automated crystallographic structure determination. Acta Crystallogr D Biol Crystallogr 58, 1948-1954 (2002).

50. M. D. Winn et al., Overview of the CCP4 suite and current developments. Acta Crystallogr D Biol Crystallogr 67, 235-242 (2011). 
51. A. Gorel, I. Schlichting, T. R. M. Barends, Discerning best practices in XFEL-based biological crystallography - standards for nonstandard experiments. Iucrj 8, 532-543 (2021).

52. P. Nogly et al., Retinal isomerization in bacteriorhodopsin captured by a femtosecond x-ray laser. Science 361, (2018).

53. P. Emsley, K. Cowtan, Coot: model-building tools for molecular graphics. Acta Crystallogr D Biol Crystallogr 60, 2126-2132 (2004).

54. B. E. Bricogne G, Brandl M, Flensburg C, Keller P, Paciorek W, Roversi P, Sharff A, Smart O, Vonrhein C, Womack T (Cambridge, United Kingdom: Global Phasing Ltd., 2021).

55. S. Salentin, S. Schreiber, V. J. Haupt, M. F. Adasme, M. Schroeder, PLIP: fully automated protein-ligand interaction profiler. Nucleic Acids Res 43, W443-447 (2015).

56. R. H. B. Smith, A. C. Dar, A. Schlessinger, PyVOL: a PyMOL plugin for visualization, comparison, and volume calculation of drug-binding sites. bioRxiv, 816702 (2019).

57. C. Slavov et al., The ultrafast reactions in the photochromic cycle of water-soluble fulgimide photoswitches. Phys Chem Chem Phys 18, 10289-10296 (2016).

58. C. Slavov, H. Hartmann, J. Wachtveitl, Implementation and evaluation of data analysis strategies for timeresolved optical spectroscopy. Anal Chem 87, 2328-2336 (2015).

59. M. P. Jacobson, R. A. Friesner, Z. Xiang, B. Honig, On the role of the crystal environment in determining protein side-chain conformations. J Mol Biol 320, 597-608 (2002).

60. G. M. Sastry, M. Adzhigirey, T. Day, R. Annabhimoju, W. Sherman, Protein and ligand preparation: parameters, protocols, and influence on virtual screening enrichments. J Comput Aided Mol Des 27, 221234 (2013).

61. H. M. A. D.A. Case, K. Belfon, I.Y. Ben-Shalom, S.R. Brozell, D.S. Cerutti, T.E. Cheatham, III, G.A. Cisneros, V.W.D. Cruzeiro, T.A. Darden, R.E. Duke, G. Giambasu, M.K. Gilson, H. Gohlke, A.W. Goetz, R. Harris, S. Izadi, S.A. Izmailov, C. Jin, K. Kasavajhala, M.C. Kaymak, E. King, A. Kovalenko, T. Kurtzman, T.S. Lee, S. LeGrand, P. Li, C. Lin, J. Liu, T. Luchko, R. Luo, M. Machado, V. Man, M. Manathunga, K.M. Merz, Y. Miao, O. Mikhailovskii, G. Monard, H. Nguyen, K.A. O’Hearn, A. Onufriev, F. Pan, S. Pantano, R. Qi, A. Rahnamoun, D.R. Roe, A. Roitberg, C. Sagui, S. Schott-Verdugo, J. Shen, C.L. Simmerling, N.R. Skrynnikov, J. Smith, J. Swails, R.C. Walker, J. Wang, H. Wei, R.M. Wolf, X. Wu, Y. Xue, D.M. York, S. Zhao, and P.A. Kollman, in University of California, San Francisco. (2021).

62. W. L. Jorgensen, J. Chandrasekhar, J. D. Madura, R. W. Impey, M. L. Klein, Comparison of Simple Potential Functions for Simulating Liquid Water. J Chem Phys 79, 926-935 (1983).

63. J. A. Maier et al., ff14SB: Improving the Accuracy of Protein Side Chain and Backbone Parameters from ff99SB. J Chem Theory Comput 11, 3696-3713 (2015).

64. O. Allner, L. Nilsson, A. Villa, Magnesium Ion-Water Coordination and Exchange in Biomolecular Simulations. J Chem Theory Comput 8, 1493-1502 (2012).

65. K. L. Meagher, L. T. Redman, H. A. Carlson, Development of polyphosphate parameters for use with the AMBER force field. J Comput Chem 24, 1016-1025 (2003).

66. R. Salomon-Ferrer, A. W. Gotz, D. Poole, S. Le Grand, R. C. Walker, Routine Microsecond Molecular Dynamics Simulations with AMBER on GPUs. 2. Explicit Solvent Particle Mesh Ewald. Journal of Chemical Theory and Computation 9, 3878-3888 (2013).

67. A. Barducci, G. Bussi, M. Parrinello, Well-tempered metadynamics: A smoothly converging and tunable free-energy method. Phys Rev Lett 100, (2008).

68. D. Van der Spoel et al., GROMACS: Fast, flexible, and free. J Comput Chem 26, 1701-1718 (2005).

69. G. A. Tribello, M. Bonomi, D. Branduardi, C. Camilloni, G. Bussi, PLUMED 2: New feathers for an old bird. Comput Phys Commun 185, 604-613 (2014).

70. D. Gobbo et al., Investigating Drug-Target Residence Time in Kinases through Enhanced Sampling Simulations. Journal of Chemical Theory and Computation 15, 4646-4659 (2019). 


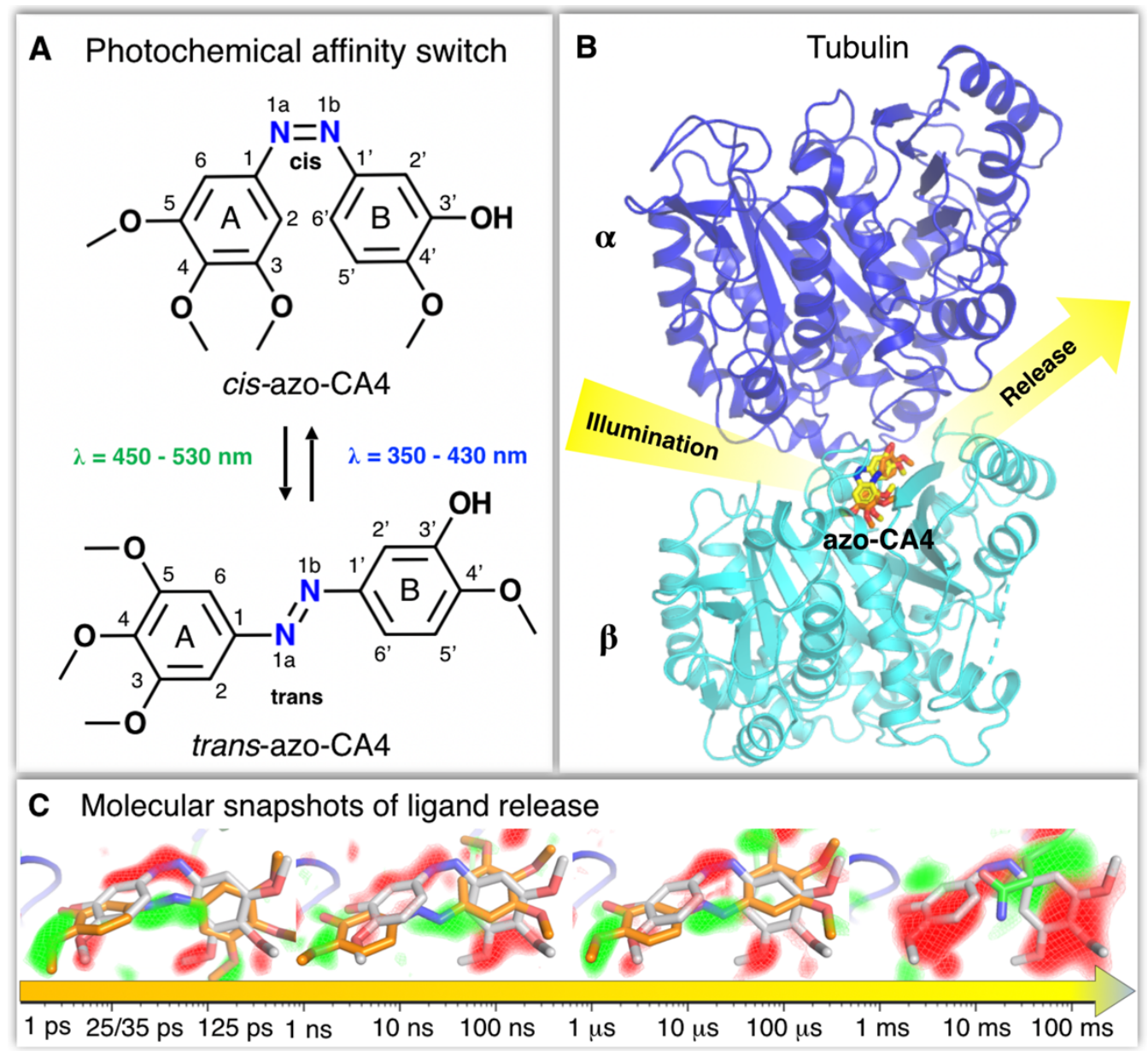

Fig. 1: Tracking drug release using a photochemical affinity switch. (A) The two chemical structures of azo-CA4 show the wavelength dependent conformational change between the cis (top) and trans (bottom) positions of the $\mathrm{A}$ and $\mathrm{B}$ rings attached to the $\mathrm{N}=\mathrm{N}$ azo bond that switches azo-CA4 between high affinity and low affinity conformations, respectively. (B) Azo-CA4 binds to the colchicine site that is formed by secondary structural elements stemming from both the $\alpha$ (blue) and $\beta$ (cyan) subunits of the $\alpha \beta$-tubulin heterodimer. (C) Thirteen molecular snapshots determined with pump-probe delays between 1 ps and $100 \mathrm{~ms}$ allow following the critical events upon azo-CA4 release. Representative difference Fourier electron difference density maps $\left(\left(\mathrm{F}_{\text {obs }}(\right.\right.$ light $)-\mathrm{F}_{\text {obs }}($ dark $)$, positive density shown as green and negative density as red mesh contoured at $3.5 \sigma$ ) for the $25 \mathrm{ps}, 100 \mathrm{~ns}, 10 \mu \mathrm{s}$ and $100 \mathrm{~ms}$ time delays are shown together with an overlay of azo-CA4 before (grey sticks) and after (orange sticks) light activation. 


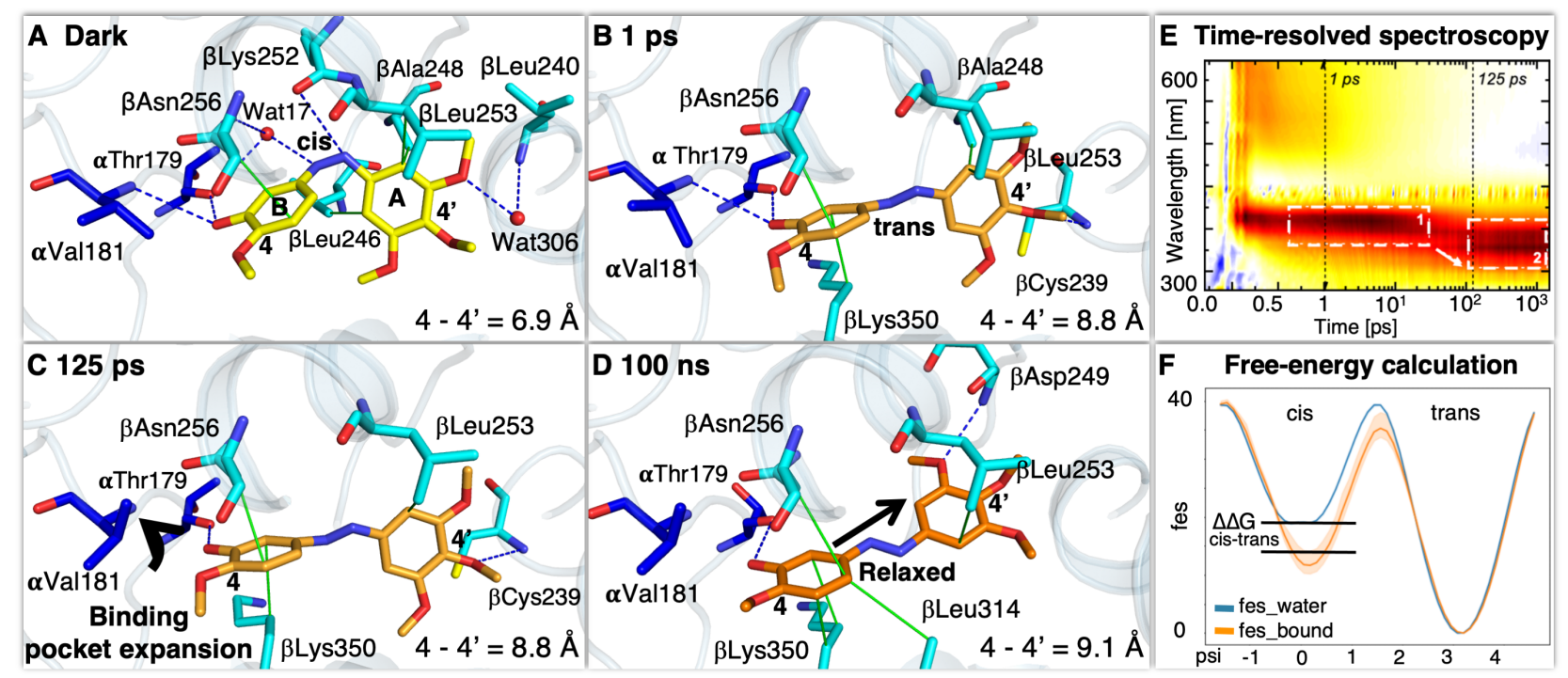

Fig. 2: Effect of the azo-CA4 isomerization. (A) Before illumination, cis-azo-CA4 is bound in the colchicine site located between the $\alpha$ (blue) and $\beta$ (cyan) subunits of tubulin. For clarity, only amino acid residues (sticks) and the two water molecules (red spheres) contacting the ligand are shown. Binding is stabilized by hydrophobic stacking and polar interactions (green lines) of tubulin residues with the $\mathrm{A}$ and $\mathrm{B}$ rings of the ligand, and by hydrophilic interactions formed by the ring substituents and the azobenzene-bond (blue dashed lines). (B) At $1 \mathrm{ps,} \mathrm{the} \mathrm{cis-trans}$ isomerization breaks the two water-mediated hydrogen bonds to the azo group and changes the stacking interactions through rotations and displacements of the two azo-CA4 rings. (C) At 125 ps, stretching of the ligand is transmitted into the protein by a push against $\alpha$ Val181 (black arrow). (D) At $100 \mathrm{~ns}$, the A ring of trans-azo-CA4 has relocated into a position closer to the $\beta \mathrm{T} 7$ loop. (E) The relaxation of the conformationally strained azo-CA4 coincides with a blue shift in the absorption maximum, which is not observed in the absence of tubulin (Fig. S3). (F) Computational analysis of the binding energy landscape provides a rationale for how cis-trans isomerization initiates ligand unbinding. The plot indicates the energy gain of the cis vs. the trans azo-CA4 isomer upon binding to tubulin. 


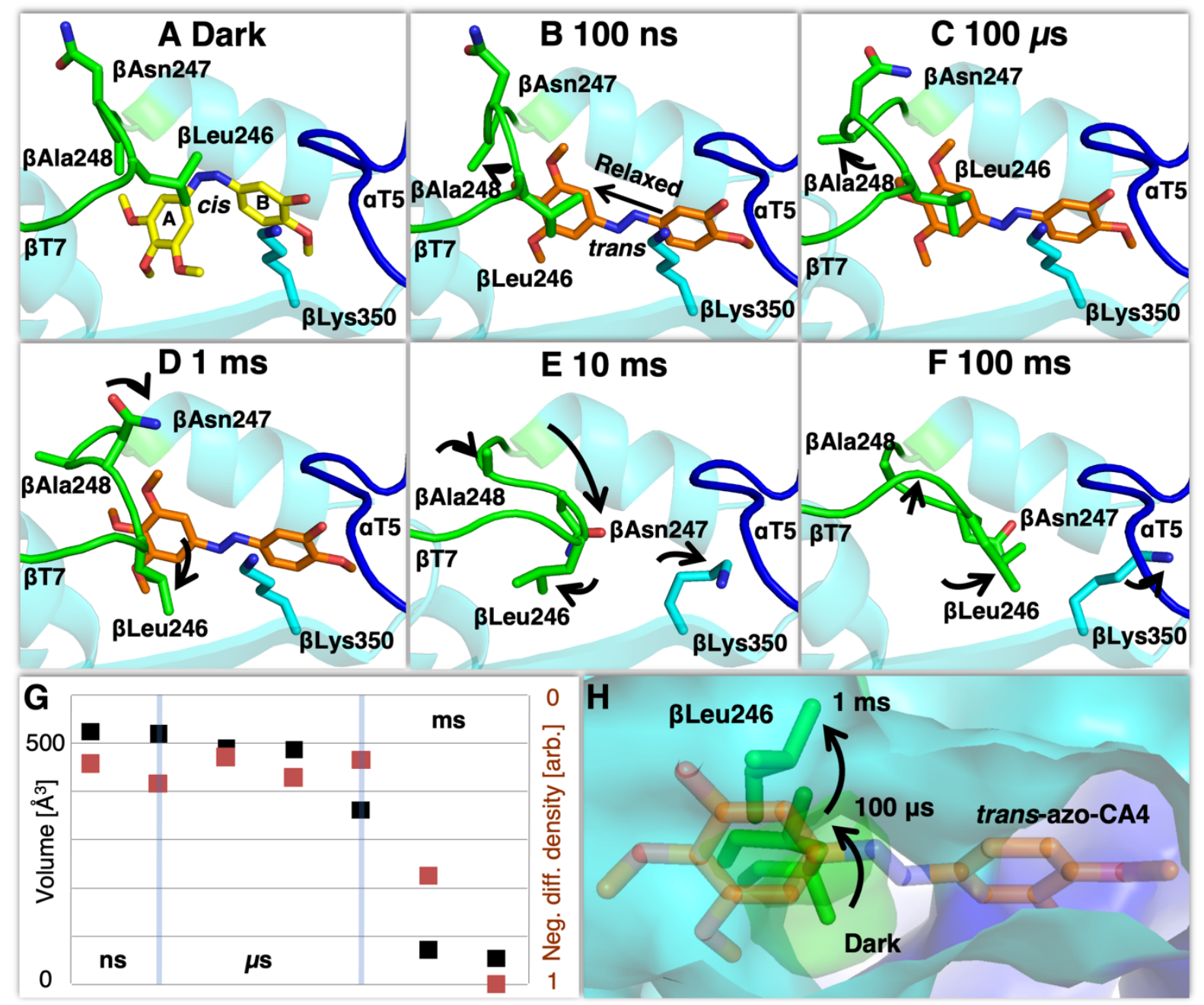

Fig. 3: Reorganization of the colchicine site upon azo-CA4 release. (A) View on cis-azo-CA4 (yellow sticks) within the colchicine site ( $\alpha$ and $\beta$-tubulin in blue and cyan, respectively). Of particular interest are the dynamics of the $\beta \mathrm{T} 7$ loop (green) connecting the $\beta \mathrm{H} 7$ and $\beta \mathrm{H} 8$ helices of $\beta$-tubulin. (B) At $100 \mathrm{~ns}$, the A ring of trans-azo-CA4 (orange sticks) is positioned closer towards the $\beta$ T7 loop. (C-D) Further reorganizations (black arrows) open a channel between the $\beta$ H8 helix and the $\beta$ T7 loop at $1 \mathrm{~ms}$. (E) At $10 \mathrm{~ms}$ after activation, the $\beta$ T7 loop has folded back into the colchicine site, where now $\beta$ Asn 247 occupies the position previously accommodating the azo-CA4 ligand. (F) Within $100 \mathrm{~ms}$ of activation, the colchicine site has collapsed and returned to its unliganded conformational state. (G) The volume of the colchicine site (black boxes) remains largely constant until the microseconds and the following collapse in the milliseconds. Between 1 and $10 \mathrm{~ms}$, the movement of the $\beta \mathrm{T} 7$ loop is followed by an increase of negative difference density $\left(\mathrm{F}_{\text {obs }}(\right.$ light $)-\mathrm{F}_{\text {obs }}($ dark $\left.)\right)$ in the original azo-CA4 position (red boxes), indicating ligand release from the binding pocket. (H) Surface representation illustrating how repositioning of the residue $\beta$ Leu246 of the $\beta$ T7 "gating loop" opens a pathway for ligand release. 

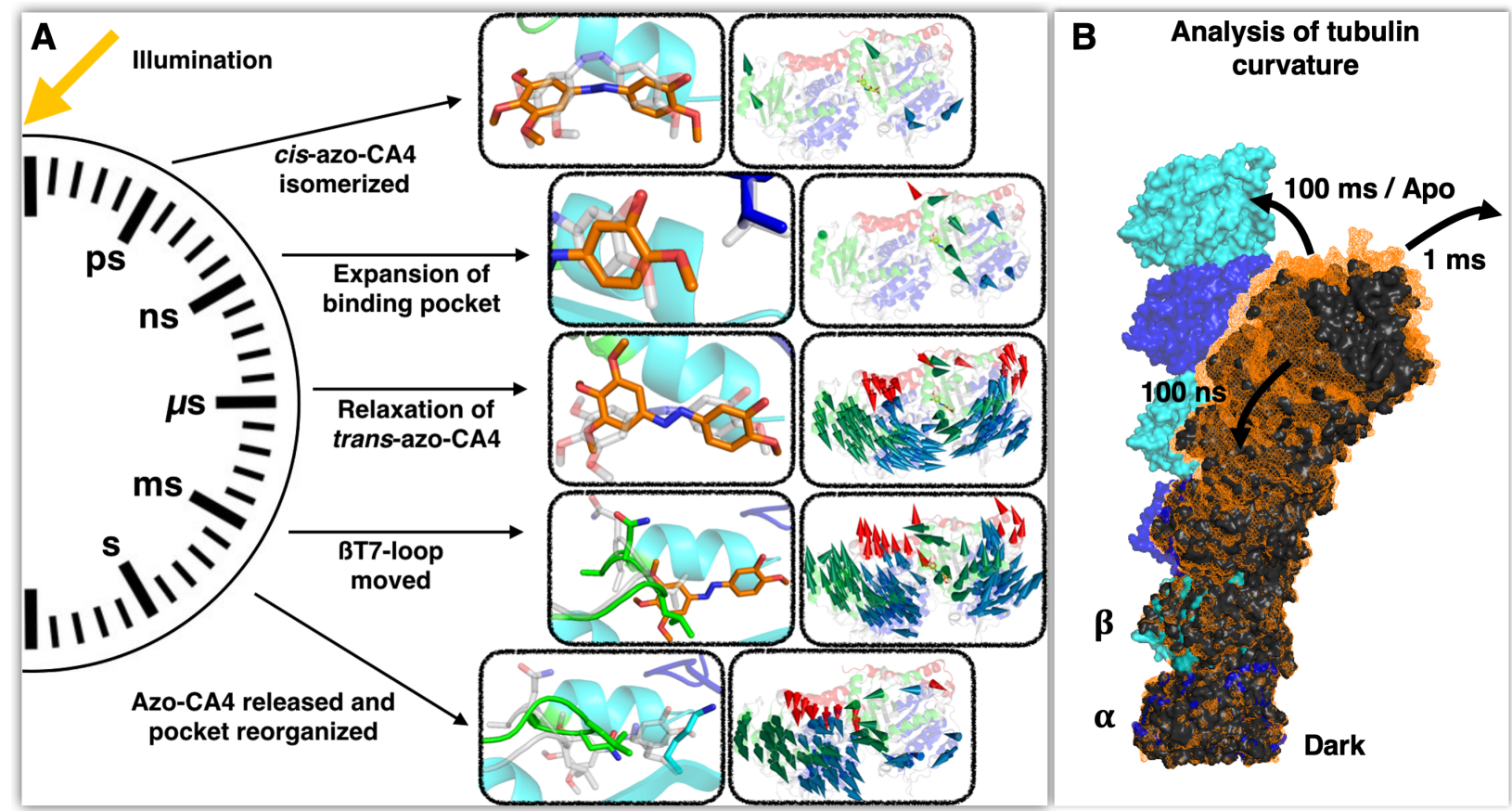

Fig. 4: Global tubulin conformational changes upon azo-CA4 release. (A) Overview of the main conformational changes (dark to $1 \mathrm{ps}, 1 \mathrm{ps}$ to $125 \mathrm{ps}, 125$ to $100 \mathrm{~ns}, 100 \mathrm{~ns}$ to $1 \mathrm{~ms}$ and $1 \mathrm{~ms}$ to $100 \mathrm{~ms}$ ) in the colchicine site and corresponding global protein backbone rearrangements. Backbone movements within tubulin are represented by modevectors between consecutive time delays. Only $\mathrm{C} \alpha$-backbone atom displacements of at least $0.5 \AA$ in the main secondary structural elements are shown (N-terminal nucleotide-binding domain in blue, C-terminal domain in red, intermediate domain in green, and core helix H8 in yellow according to (38)). (B) Comparison of the conformational states of "straight" tubulin ( $\alpha$ - and $\beta$-tubulin in blue and cyan surface representations; PDB ID 5SYE (39)), and "curved" tubulin before (black) and at selected time delays after light activation (orange). Shown are three longitudinally aligned tubulin dimers. Arrows indicate the main direction of movements at the indicated time delays. The final release of azo-CA4 reduces the curvature of the three tubulin dimers by $2.6^{\circ}$ in the direction of the straight protofilament conformation found in a microtubule (30) (see also Fig. S6). 


\section{Supplementary Materials for}

\section{Molecular snapshots of drug release from tubulin over eleven orders of magnitude in time}

Maximillian Wranik ${ }^{1}$, Tobias Weinert ${ }^{1}$, Chavdar Slavov², Tiziana Masini ${ }^{3}$, Antonia Furrer ${ }^{1}$, Natacha Gaillard $^{1}$, Dario Gioia ${ }^{3}$, Marco Ferrarotti ${ }^{3}$, Daniel James ${ }^{1}$, Hannah Glover ${ }^{1}$, Melissa Carrillo ${ }^{1}$, Demet Kekilli $^{1}$, Robin Stipp ${ }^{1}$, Petr Skopintsev ${ }^{1}$, Steffen Brünle ${ }^{1}$, Tobias Mühlethaler ${ }^{1}$, John Beale ${ }^{1}$, Dardan Gashi ${ }^{4}$, Karol Nass ${ }^{4}$, Dmitry Ozerov ${ }^{5}$, Philip J.M. Johnson ${ }^{4}$, Claudio Cirelli ${ }^{4}$, Camila Bacellar ${ }^{4}$, Markus Braun ${ }^{2}$, Meitian Wang ${ }^{4}$, Florian Dworkowski ${ }^{4}$, Chris Milne ${ }^{4}$, Andrea Cavalli ${ }^{3,6}$, Josef Wachtveitl ${ }^{2}$, Michel O. Steinmetz ${ }^{1,7, *}$ Jörg Standfuss ${ }^{1, *}$

${ }^{1}$ Division of Biology and Chemistry, Paul Scherrer Institut, 5232 Villigen, Switzerland.

${ }^{2}$ Institute of Physical and Theoretical Chemistry, Goethe University, Frankfurt am Main, Germany.

${ }^{3}$ Computational \& Chemical Biology, Istituto Italiano di Tecnologia, 16163 Genova, Italy.

${ }^{4}$ Photon Science Division, Paul Scherrer Institut, 5232 Villigen, Switzerland.

${ }^{5}$ Scientific Computing, Theory and Data, Paul Scherrer Institut, 5232 Villigen, Switzerland.

${ }^{6}$ Department of Pharmacy and Biotechnology, University of Bologna, 40126 Bologna, Italy.

${ }^{7}$ Biozentrum, University of Basel, 4056 Basel, Switzerland.

*Correspondence to: michel.steinmetz@psi.ch and joerg.standfuss@psi.ch

This PDF file includes:

Materials and Methods

Supplementary Text

Figs. S1 to S6

Tables $\mathrm{S} 1$ to $\mathrm{S} 2$

Captions for Movies S1 to S2

Other Supplementary Materials for this manuscript include the following:

Movies S1 to S2 


\section{Materials and Methods}

\section{Tubulin purification and crystallization}

Bovine brain tubulin was purchased from the Centro de Investigaciones Biológicas (Microtubule Stabilizing Agents Group), CSIC, Madrid, Spain. The DARPin D1 construct was adapted, expressed and purified according to previous descriptions (16). The tubulin-DARPin D1 (TD1) complex was formed by mixing the respective components in a 1:1.1 molar ratio. The TD1 complex was crystallized on EasyXtal 15-well plates (QIAGEN) by the hanging drop vapor diffusion method (drop size $2 \mu \mathrm{L}$, drop ratio 1:1, 8 drops per well) at a concentration of $9.8 \mathrm{mg}$ $\mathrm{mL}^{-1}$ at $20^{\circ} \mathrm{C}$ with a precipitant solution containing $21 \%(\mathrm{w} / \mathrm{v})$ PEG 3000, $0.2 \mathrm{M}$ ammonium sulfate and $0.1 \mathrm{M}$ bis-tris methane, $\mathrm{pH}$ 5.5. All drops were subsequently hair-seeded with crystalline material obtained in previous crystallization attempts to increase homogeneity and density of crystals. After 48 hours, crystals were washed off the plates with precipitant solution, collected in $0.6 \mathrm{ml}$ tubes and vortexed for a few seconds. This procedure induces a batchcrystallization within the tubes and a sedimented crystal pellet after 24 hours of incubation at $20^{\circ} \mathrm{C}$. Initially crystals grew as long needles that could be broken into smaller fragments of approximately $15 \times 5 \times 5 \mathrm{~m}^{3}$ during the sample preparation described below.

\section{Azo-combretastatin A4 synthesis}

Azo-CA4 (compound 6) was prepared and synthesized following the experimental procedure described in detail by Borowiak et al (11), according to the following synthetic scheme:

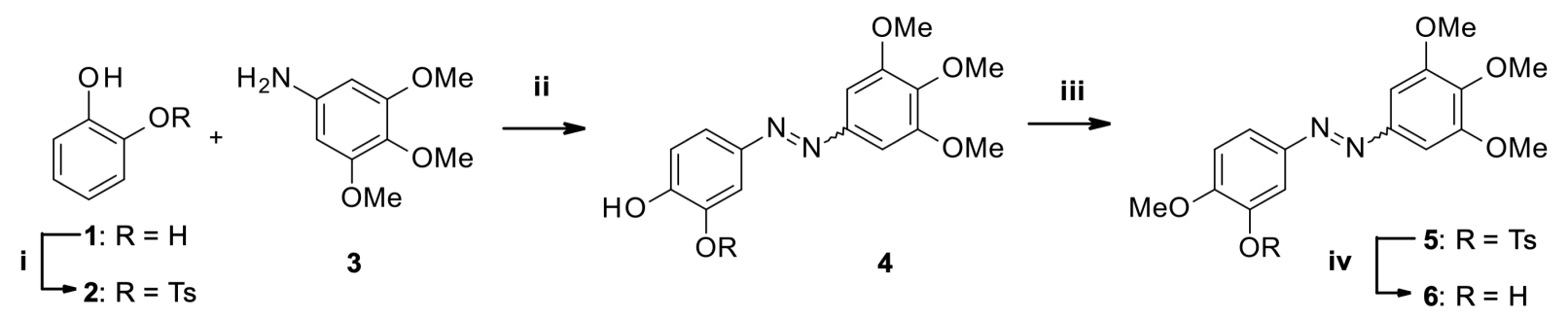

i. TsCl, Pyridine, r.t., 24 h, yield $70 \%$. ii. isopentyl nitrite, $\mathrm{HCl}$ conc., $\mathrm{NaOH}(2 \mathrm{M}), \mathrm{MeOH}, 0^{\circ} \mathrm{C}$., 5 h, yield $66 \%$. iii. $\mathrm{MeI}, \mathrm{K}_{2} \mathrm{CO}_{3}$, acetone, r.t., 16 h, yield $99 \%$. iv. $\mathrm{KOH}, \mathrm{MeOH}$, reflux, 1 h, yield $78 \%$.

Following mono-tosylation of catechol 1, the resulting intermediate 2 was subjected to diazo coupling with 3,4,5trimethoxy aniline (3) using isopentyl nitrite in methanol, leading to $\mathbf{4}$ as a mixture of $\mathrm{E} / \mathrm{Z}$ isomers $(1 / 0.8)$. Diazocompound $\mathbf{4}$ was then methylated in acetone leading to intermediate $\mathbf{5}$, and the tosyl protecting group was finally removed in basic media to deliver azo-CA4, compound $\mathbf{6}$, as a mixture of $\mathrm{E}$ and $\mathrm{Z}$ isomers. 


\section{Sample preparation serial crystallography}

Crystal pellets were resuspended and the contents of two $0.6 \mathrm{~mL}$ tubes were combined and collected into $2 \mathrm{~mL}$ tubes. After centrifugation in a tabletop centrifuge (2000 rpm, 15 seconds), a concentrated crystal pellet of 45-60 $\mu \mathrm{L}$ volume was obtained. The pellets were extracted, transferred into PCR tubes and mixed with the trans-azo-CA4 to a final concentration of $1 \mathrm{mM}$.

To switch the compound into the binding cis conformation, the tubes were then illuminated for at least 4 hours at $385 \mathrm{~nm}$ before incorporation into hydroxyethylcellulose hydrogel (Sigma-Aldrich) (40). Hydrogels containing $22 \%(\mathrm{w} / \mathrm{v})$ hydroxyethylcellulose were prepared in syringes and left to cure for 5 days. Illuminated crystals were transferred into a Hamilton syringe and mixed into hydrogel matrix at a ratio of 4:7 using a three-way-coupler (41) before loading into the high viscosity injector.

\section{Experimental setup and XFEL data collection}

Time-resolved serial crystallographic data of light-induced azo-CA4 from tubulin were collected within 4 days of beamtime in September 2020 at the Alvra experimental station of SwissFEL. The $\mathrm{X}$-ray source provided pulses with a photon energy of $12.1 \mathrm{KeV}$ and a pulse energy between 300 $\mu \mathrm{J}$ and $550 \mu \mathrm{J}$ at a repetition rate of $100 \mathrm{~Hz}$. To reduce X-ray scattering (background level), the sample chamber was pumped down to a pressure of $150 \mathrm{mbar}$ and then refilled with helium to 500 mbar. This process was continuously repeated during the measurement, except for the 1 ps time delay where pressure changes would have had a significant impact on timing accuracy. Data were collected using a Jungfrau $16 \mathrm{M}$ operated in $4 \mathrm{M}$ mode. Hit rate determination and initial data processing during the experiment were done with the online data analysis pipeline described by Nass et al. (42).

TD1 crystals homogeneously incorporated into Hydroxyethylcellulose were loaded into a high viscosity injector connected to an HPLC pump (43). The system extruded the crystal loaded hydrogel matrix into the pump-probe interaction point through a $75 \mu \mathrm{m}$ capillary at a flow rate of $5.3 \mu \mathrm{l}$ per minute. In the interaction point, the probing XFEL beam intersected with a circularly polarized pump beam originating from an optical parametric amplifier producing laser pulses with $350 \mathrm{fs}$ duration $\left(1 / \mathrm{e}^{2}\right), 475 \mathrm{~nm}$ wavelength and $10 \mu \mathrm{J}$ total energy in a focal spot of $65 \mu \mathrm{m}\left(1 / \mathrm{e}^{2}\right)$ diameter; corresponding to a maximal laser fluence of $301 \mathrm{~mJ} / \mathrm{cm}^{2}$ and laser power density of 861 $\mathrm{GW} / \mathrm{cm}^{2}$. In the 1 ps time delay a laser pulse with $150 \mathrm{fs}$ duration $\left(1 / \mathrm{e}^{2}\right)$ was used while keeping the other parameters the same. In order to follow the complete reaction of light-induced azo-CA4 
ligand release from tubulin between the pump laser and the probing, XFEL pulses were chosen at $\Delta \mathrm{t}=1 \mathrm{ps}, 25 \mathrm{ps}, 35 \mathrm{ps}, 125 \mathrm{ps}, 1 \mathrm{~ns}, 10 \mathrm{~ns}, 100 \mathrm{~ns}, 1 \mu \mathrm{s}, 10 \mu \mathrm{s}, 100 \mu \mathrm{s}, 1 \mathrm{~ms}$ and $10 \mathrm{~ms}$.

\section{Serial synchrotron crystallography}

Serial synchrotron data were collected using a previously described setup (19). Crystals embedded into hydroxyethylcellulose were injected in the intersecting X-ray and laser beam paths by a high viscosity injector (43) with a $75 \mu \mathrm{m}$ diameter nozzle.

The jet speed was set to $0.5 \mu \mathrm{m} / \mathrm{ms}$ and data frames were recorded using an Eiger $16 \mathrm{M}$ detector with a frame rate of $100 \mathrm{~Hz}$. The beam size was set to $20 \times 5 \mu \mathrm{m}^{2}$ to cover the central part of the hydroxyethylcellulose column on one axis and extrusion by one frame on the other. In this way we ensured the majority of crystals were exposed, whilst minimizing similarities in consecutive frames for more reliable statistics. A photon flux of around $1.5 \times 10^{12}$ photons $/ \mathrm{s}$ resulted in a radiation dose of about $5 \mathrm{kGy}$ per recorded detector frame when calculated with RADDOSE-3D (44). Depending on their size and orientation while traversing the beam this corresponds to between 5 and maximally $100 \mathrm{kGy}$ per crystal. Illumination was done using light at $445 \mathrm{~nm}$ from a laser diode with a peak optical power of $5 \mathrm{~mW}$ (Roithner Lasertechnik). Light intensity at the interaction region was approximately $2.5 \mathrm{~mW}$ in a spot size of about $100 \mathrm{x} 50 \mu \mathrm{m}^{2}$. The illumination time was set to approximately $100 \mathrm{~ms}$ by adjusting the distance between the laser and $\mathrm{X}$-ray interaction regions.

\section{Data processing}

Indexing, integrating and merging of obtained data was performed using Crystfel version 0.9.1 $(45,46)$. In detail, the peakfinder9 algorithm was used for peak detection, xgandalf $(47)$ was used to index obtained data; and peaks were integrated using --ring-radius $=2,3,6 ;$ partialator options -model=unity, --iterations=1.0, --push-res=1.5 were used to merge selected patterns. A general resolution cutoff of $1.7 \AA$ was chosen (see Table S1) and refinements for collected dark-state and apo-state data were carried out to the full resolution range. Structural refinements of time-resolved data were carried out to $2.2 \AA$ resolution due to data degradation when extrapolating data (see Table S1). Serial synchrotron crystallography data obtained at SLS showed stronger anisotropy than the data obtained at the SwissFEL. The data were therefore corrected for anisotropy using the STARANISO server (48). 


\section{Difference density maps calculation}

Calculations of $\mathrm{F}_{\mathrm{obs}}($ light $)-\mathrm{F}_{\mathrm{obs}}($ dark) difference maps were performed using PHENIX (49). In detail, the multi-scaling option was used excluding amplitudes smaller than $2 \sigma$ and exclusively considering the resolution range between $6 \AA$ and $1.7 \AA$. For all calculated $\mathrm{F}_{\text {obs }}($ light $)-\mathrm{F}_{\text {obs }}($ dark $)$ difference maps, the phases of the refined dark state were used. $F_{\text {calc }}($ light $)-F_{\text {calc }}($ dark $)$ difference maps were computed to the same resolution using the CCP4 suite (50).

\section{Pearson correlation of difference maps}

To compute Pearson correlations independent of individual dataset multiplicities we reduced the number of patterns to match the time delay where we collected the least data $(55,065$ patterns) and calculated difference maps as stated above. These difference maps where then used to calculate the Pearson correlation of integrated difference electron densities (20) around all protein atoms and around the ligand and all residues lying within a $3 \AA$ sphere around the ligand. Water molecules and hydrogen atoms were excluded in the selection. The density maps were integrated within a 2 $\AA$ radius around the atoms and densities below $1.5 \sigma$ where ignored. The resulting Pearson correlation values where normalized to the largest correlation, displayed on a gray scale and values smaller than 0.3 were omitted from the figures for clarity.

\section{Data extrapolation}

Calculation of extrapolated data was performed using the method described by Pandey et al. (21). Briefly, the linear approximation was used: $F_{\text {extra }}=\left[100 / A \times\left(F_{\text {obs }}(\right.\right.$ light $)-F_{\text {obs }}($ dark $\left.)\right]+F_{\text {calc }}$, with A representing the activation level in percent, $F_{\text {calc }}$ representing amplitudes of the high-resolution dark state model and $\mathrm{F}_{\text {extra }}$ representing the extrapolated structure factor amplitude. The activation level A, defined as the percentage of dark state molecules being activated after pump laser pulse exposure, was determined as described (21). Briefly, extrapolated data were calculated using a decreasing activation level $\mathrm{A}$. Then the negative electron density in $2 \mathrm{~F}_{\text {extra }}-\mathrm{F}_{\text {calc }}$ maps calculated using the dark model phases, was integrated around nitrogen atoms of the ligand which have strong negative difference density peaks on them in each of the light activated structures and hence leave their position. The negative density was then plotted against the activation level. The intersection of the linear parts of the resulting graph (negative density is zero for not enough dark state subtracted and starts to linearly increase as dark is over subtracted) was taken as the activation level. The activation levels determined in this way fluctuated between $18-27 \%$ but since all data 
(except the 1 ps time delay) were measured under the same conditions we chose the average of $22 \%$ to refine the structures. As a further control we determined the activation level using the reappearance of dark state features in $2 \mathrm{~F}_{\text {extra }}-\mathrm{F}_{\text {calc }}$ maps $(51,52)$, which confirmed our initial assignment.

\section{Structure determination and refinement}

The structure of the dark-state was solved by molecular replacement with 5NQT as a search model. Structural refinements of the model were done using PHENIX (49) with iterative cycles of manual adjustments made in Coot (53). As a final step the ligand was refined using Buster (54), employing the -qm option to use a quantum mechanics target for the ligand rather than restraints which may be a source of errors, especially for molecules which are not at their energy minimum. The structure of the apo-state was directly refined using the final dark-state structure as a starting point. Before the actual refinement and model building of the obtained time-resolved data, the negative amplitudes resulting from the extrapolation procedure were removed. The models were manually adjusted to best fit observed difference map features as well as extrapolated maps using the final dark-state model as a starting point.

Before the actual refinement and model building of the obtained time-resolved data, the negative amplitudes resulting from the extrapolation procedure were removed. The models were manually adjusted to best match observed difference map features, to produce similar $F_{\text {calc }}($ light $)-F_{\text {calc }}($ dark) maps and to fit extrapolated maps using the final dark-state model as a starting point. To match the observed difference density features using $\mathrm{F}_{\text {calc }}(1 \mathrm{~ms})-\mathrm{F}_{\text {calc }}$ (dark) maps we chose to exclude Leu 246 from XYZ refinement after manual adjustment for the $1 \mathrm{~ms}$ structure (See Figure S2). Given the activation level of 22 percent we choose to refine the predominant structural species by comparing the models to previous and consecutive structures.

\section{Binding pocket and protein backbone analysis}

Protein interactions in the binding pocket were identified with the protein-ligand interaction profiler (55). Changes in the volume of the colchicine binding site over time were determined using PyVOL (50) with the parameters (minimum radius $=1.65$; maximum radius $=3.8$; minimum volume $=50$, standard partitioning)

To visualize global protein backbone movements, alternative conformers were removed and structures truncated to poly-Ala using Phenix PDB tools (49). Respective structural displacements 
of secondary structural elements were indicated using the PyMOL Python script Modevectors (by Sean M. Law, University of Michigan). Displacements greater than $0.5 \AA$ were classified as significant (cutoff $=0.5$ ). For visualization purposes, only the displacement vectors of every second $($ skip=2) a-backbone atom (atom=CA) were shown and the vectors amplified by a factor of 6 (factor=6). Additional settings involve the vector representation (head=2.0, tail $=0.85$, head_length $=3.0$, cut $=0)$.

\section{Time-resolved spectroscopy measurements}

The time-resolved transient absorption data were recorded using a home-built pump-probe setup, as described in detail previously (57). In short, the fundamental laser pulses ( $1 \mathrm{~mJ}, 775 \mathrm{~nm}, 130$ fs, $1 \mathrm{kHz}$ ) were provided by a Ti:Sa amplifier (Clark, MXR-CPA-iSeries). The pump pulses were generated using a home-built two stage NOPA (noncollinear optical parametric amplifier) and sum frequency mixing. The pulses were pre-compressed in a prism compressor located between the two NOPA stages. White light continuum pulses $(300-750 \mathrm{~nm})$ were generated by focusing a fraction of the laser fundamental beam into a $\mathrm{CaF}_{2}$-crystal $(5 \mathrm{~mm})$. The continuum pulses were split into probe and reference beams. The reference beam was guided directly into a spectrograph, while the probe beam was focused at the sample position then collected and directed into a second spectrograph. The spectrographs (AMKO Multimode) contained gratings with 600 grooves $/ \mathrm{mm}$ blazed at $300 \mathrm{~nm}$ and a photodiode array. The instrument response function (IRF) of $\sim 100 \mathrm{fs}$ in the experiments was estimated from the pump probe cross correlation. Anisotropic contributions to the measurements were avoided by performing the experiments at the magic angle condition (54.7 $7^{\circ}$ pump-probe polarization difference). The samples were held in a fused silica cuvette with an optical path length of $1 \mathrm{~mm}$ which was constantly moved in the plane perpendicular to the direction of the probe pulse propagation to avoid accumulation of photoproducts. To keep the azoCA4 samples (bound and free) in the cis isomer state, the cuvette was continuously illuminated with a high-power LED at $385 \mathrm{~nm}$. Global analysis of the experimental data was performed using OPTIMUS (www.optimusfit.org) (58) to recover the lifetimes describing the cis $\rightarrow$ trans photoisomerization kinetics. With the help of target analysis, a sequential kinetic scheme was fitted to the experimental data to obtain the times of the highest population of each kinetic state, which were helpful in determining the optimal time points for the XFEL experiments. 


\section{Computational Methods}

\section{System preparation}

The starting models for simulations were built from the respective XFEL-based time-resolved structures. We removed the DARPin protein and reconstructed the $\beta$ M-loop (res 272-288) using the one in chain D of PDB 4I4T (tubulin-RB3-TTL-Zampanolide complex) as template by employing the Prime (59) module of the Schrödinger 2020-4 suite. Analogously, $\mathrm{Mg}^{2+}$ ion in chain B ( $\beta$-tubulin) was added by superimposition with the one of chain D of 4I4T structure. Calcium ions were removed from the model.

The final model consisted of an $\alpha / \beta$-tubulin heterodimer in complex with azo-CA4, GTP and GDP molecules bound to the $\alpha$ - and $\beta$-tubulin monomers, respectively, as well as their associated $\mathrm{Mg}^{2+}$ ions and their coordinating water molecules. The resulting protein structure possessed 437 out of 451 residues of $\alpha$-tubulin (UniProtKB ID P81947) and 431 out of 445 residues of $\beta$-tubulin (UniProtKB ID Q6B856). Missing residues belonging to the intrinsically disordered C-terminal tails of $\alpha$ - and $\beta$-tubulin were not modeled and C-termini were capped with $\mathrm{N}$-methyl amide (NME) groups. Residue protonation states were evaluated at $\mathrm{pH} 7.0$ using the Protein Preparation Wizard tool (60) implemented in the Schrödinger 2020-4 suite (61). The $\alpha \beta$-tubulin heterodimer structure was solvated with the TIP3P-model (62) for water molecules in a truncated octahedron box using $12 \AA$ as minimum distance between the protein and the box edges. The system was neutralized by adding $\mathrm{Na}^{+}$ions resulting in a total of about $122 \mathrm{k}$ atoms. The atomistic force field Amber-ff14SB (63) was used for all simulations. Parameters for $\mathrm{Mg}^{2+}$ ions and the GTP and GDP molecules were developed by Allner et al. (64) and Meagher et al. (65) respectively.

Concerning the ligand, partial atomic charges were obtained with the Restrained Electrostatic Potential (RESP) method on a DFT-optimized trans conformation at the B3LYP/6-31g* level. The geometric optimization was carried out with Terachem v1.94. The General Amber ForceField (GAFF) was used, with custom parameters for the three main dihedral angles: 1-1a-1b-1', 6-1-1a1b, 1a-1b-1'-6'.

In order to obtain such optimized parameters, we fitted the GAFF energy curves associated with a rigid torsion of the dihedral angles to the corresponding DFT energy curves. For each of the considered dihedral we proceeded as follows: we started out with the geometrically optimized trans structure. We then performed a clockwise rigid rotation with $15^{\circ}$ steps followed by an analogous counter-clockwise rigid rotation. For each rotation step we performed a DFT geometric optimization restraining the rotated dihedral and we evaluated the energy. We retained the 
minimum energy among the clockwise and anticlockwise rotation cycles to overcome hysteresis. The three resulting DFT energy profiles consisting of 24 evaluation points were used as a target for the fitting procedure. DFT calculations were performed with Terachem v1.94, at the B3LYP/6$31 \mathrm{~g}^{*}$ level. The fitting procedure was carried out separately for the three energy profiles with mdgx, part of AmberTools21. The $\alpha \beta$-tubulin heterodimer system was assembled with the LEaP tool implemented in the AmberTools 21 software package (61).

The designed models for QM calculations consisted of protein residues $\alpha(99-101,178-181), \beta(237$ 242, 248-259, 314-321, 349-354, 378-380), with the respective N- and C- termini capped with acetyl (ACE) and NME groups, the azo-AC4 molecule, and its two closest water molecules. They resulted in 743 atoms.

\section{MD simulations}

Plain MD simulations starting from the trans azo-AC-tubulin complex $1 \mathrm{~ms}$ structure were performed using the Amber20 engine (61). The system, after a preliminary stage of energy minimization (1000 steps of steepest descent and 9000 of conjugate gradient), was heated to the target temperature of $300 \mathrm{~K}$ with a $250 \mathrm{ps}$ ramp using an integration time-step of $1 \mathrm{ps}$ and the weak-coupling algorithm, followed by another 500 ps of simulation in the NVT ensemble after switching to an integration time-step of 2 ps. Subsequently, in order to the equilibrate box dimensions, the system was simulated for $1 \mathrm{~ns}$ in the NPT ensemble using the Berendsen barostat. For these initial stages, heavy atoms were harmonically restrained with a force constant of 5 $\mathrm{kcal} / \mathrm{mol} / \AA^{2}$. Finally, a last stage of $5 \mathrm{~ns}$ in the NVT ensemble, using the Langevin thermostat with a collision frequency $\gamma$ of $2.0 \mathrm{ps}^{-1}$. Bonds involving hydrogen atoms were restrained, a short-range, non-bonded cut-off of $8 \AA$ was applied, whereas long-range electrostatics were treated with the particle mesh Ewald (PME) method (66). Periodic boundary conditions (PBC) were applied. After the equilibration stage, a cumulative $30 \mu$ s-long MD simulation was conducted with an integration time step of $2 \mathrm{fs}$ in the NVT ensemble at a target temperature of $300 \mathrm{~K}$.

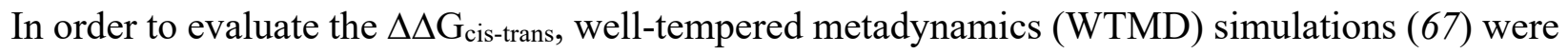
performed with GROMACS 2020.2 (68) and PLUMED 2.6.1 (69). We performed WTMD enhancing the sampling of the two dihedral angles 1-1a-1b-1' and 6-1-1a-1b for both the bound and the unbound state. The bias factor, the deposition rate, the initial hill height, and the hill width were set to $45,0.5 \mathrm{ps}^{-1}, 2.5 \mathrm{~kJ} \cdot \mathrm{mol}^{-1}$, and $0.3 \mathrm{rad}$, respectively. 
The WTMD simulation of the bound state was carefully designed to sample conformations compatible to the XFEL-based time-resolved structures. A positional harmonic restraint with $\mathrm{K}=100 \mathrm{~kJ} / \mathrm{mol} \mathrm{nm}^{\wedge} 2$ was introduced on the backbone of the protein together with the supervision of the hydrogen bond to the backbone carbonyl of $\alpha$ Thr179. With this regard, the simulation was stopped and restarted with regenerated velocities whenever the H-bond was broken. The resulting trajectory of 400 ns was reweighted in order to reconstruct the free energy profile shown in Figure 2(G). Block analysis with block size of $50 \mathrm{~ns}$ was performed to assess convergence and to plot 95\% confidence interval error bands.

The WTMD simulation of the unbound state was performed with the same parameters used for the bound state. In this case, we simulated a single ligand molecule in a solvated cubic box using 10 $\AA$ as minimum distance between the ligand and the box edges. After standard minimization, thermalization and equilibration phases WTMD was carried out for $400 \mathrm{~ns}$. The resulting trajectory was reweighted in order to reconstruct the free energy profile shown in Figure 2 G. Block analysis with block size of $50 \mathrm{~ns}$ was performed to assess convergence and to plot $95 \%$ confidence interval error bands. 
Binding pocket

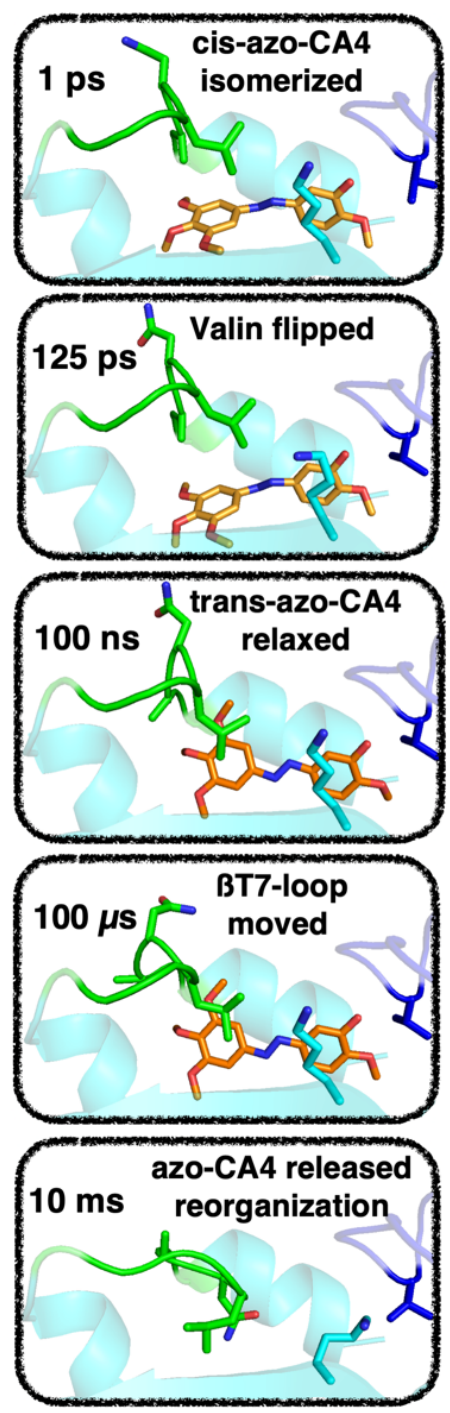

Backbone movements
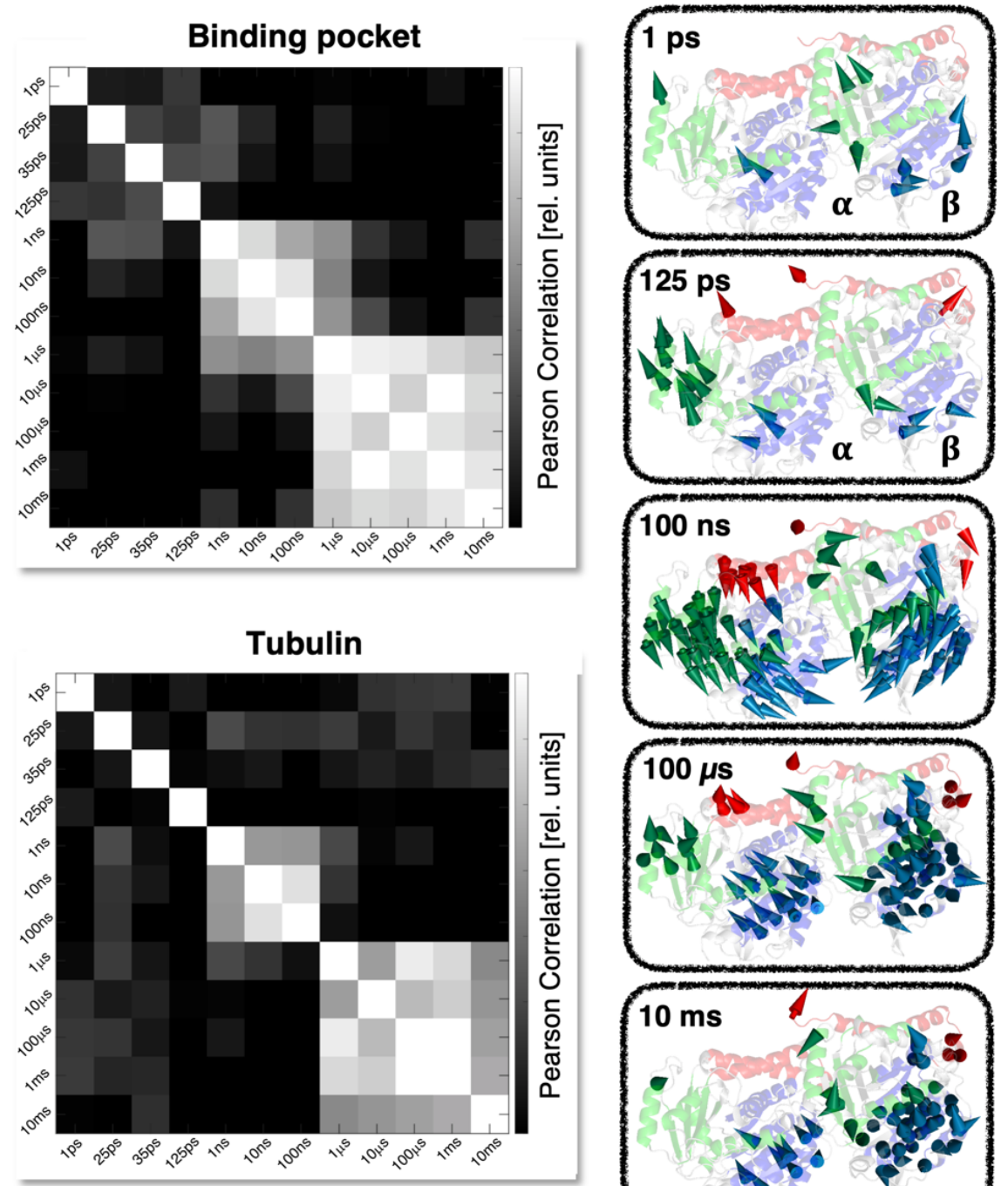
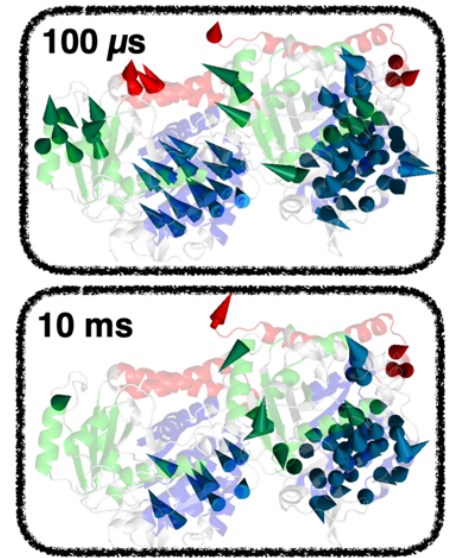

Fig. S1: Pearson correlation of difference electron density changes over time. Electron density maps $\left(\mathrm{F}_{\text {obs }}(\right.$ light $)-\mathrm{F}_{\text {obs }}($ dark $)$ ) were integrated around atoms in the colchicine site (upper middle panel) or the whole tubulin-azo-CA4 complex (lower middle panel) and subjected to Pearson correlation analysis (20). The $100 \mathrm{~ms}$ data that were collected at the SLS has not been included because differences in activation level and resolution prevent a direct comparison on the electron density level. Relevant structural changes on representative time points are shown for the ligandbinding pocket (left panels) and the whole protein (right panels). Global protein backbone movements are represented as modevectors relative to the dark structure. Only C $\alpha$-backbone atom displacements of at least $0.5 \AA$ in major secondary structural elements of $\alpha$ - and $\beta$-tubulin are shown (N-terminal nucleotide-binding domain in blue, $\mathrm{C}$-terminal domain in red, intermediate domain in green according to (38)); their magnitudes were multiplied by a factor of six for better visualization. 


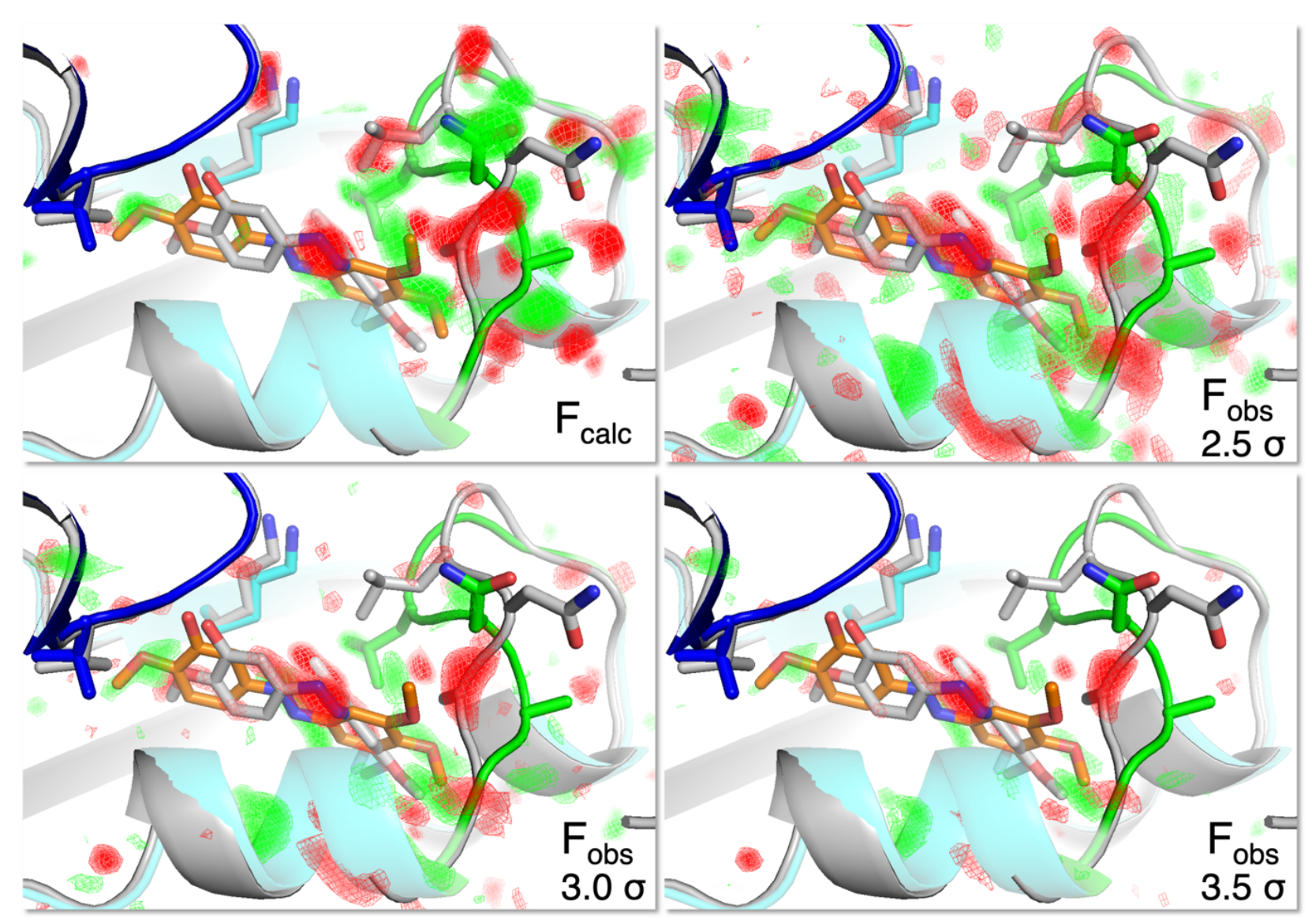

Fig. S2: Comparison of $\mathbf{F}_{\text {obs }}$ and $\mathbf{F}_{\text {calc }}$ difference maps. Experimentally observed $\mathrm{F}_{\mathrm{obs}}(1 \mathrm{~ms})$ $\mathrm{F}_{\text {obs }}($ dark $)$ and calculated $\mathrm{F}_{\text {calc }}(1 \mathrm{~ms}-$ model $)-\mathrm{F}_{\text {calc }}($ dark-model) difference density maps within the ligand binding site are shown. Positive and negative difference density are displayed in red and green, respectively. The initial dark model is shown in grey and the $1 \mathrm{~ms}$ structure as blue $(\alpha$ tubulin), cyan ( $\beta$ tubulin) and green ( $\beta$ T7 loop). Selected side chains are displayed in stick representation. The $\mathrm{F}_{\text {calc }}$ difference density map (top) was calculated using B-factors of 30 for all atoms and is displayed at a sigma level of 3.5 sigma, while the $F_{\text {obs }}$ maps are shown at levels from 2.5 to 3.5 sigma. The $\mathrm{F}_{\text {calc }}$ maps represent a full activation level and data without noise and even though this prevents direct comparison of sigma levels, there is an excellent agreement between the simulated and calculated difference maps. 

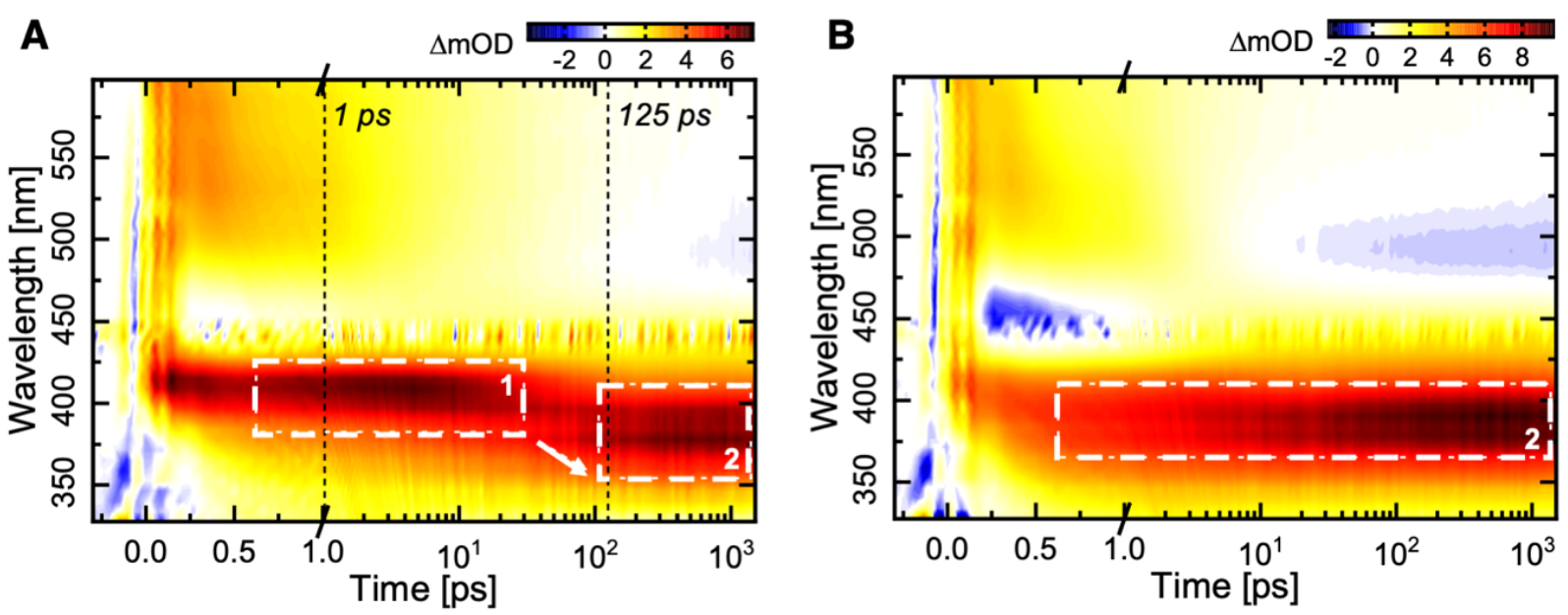

Fig. S3: Time-resolved spectroscopic data collected in the absence and presence of tubulin. Between $1 \mathrm{ps}$ and $125 \mathrm{ps}$, the spectrum of tubulin-bound azo-CA4 (A) shows a transition from the primary strained trans-isomer to a conformationally relaxed trans-isomer as reflected by the shift of the corresponding absorption to shorter wavelengths (indicated by rectangles 1 and 2). Such a shift is not observed in the absence of tubulin (B). 


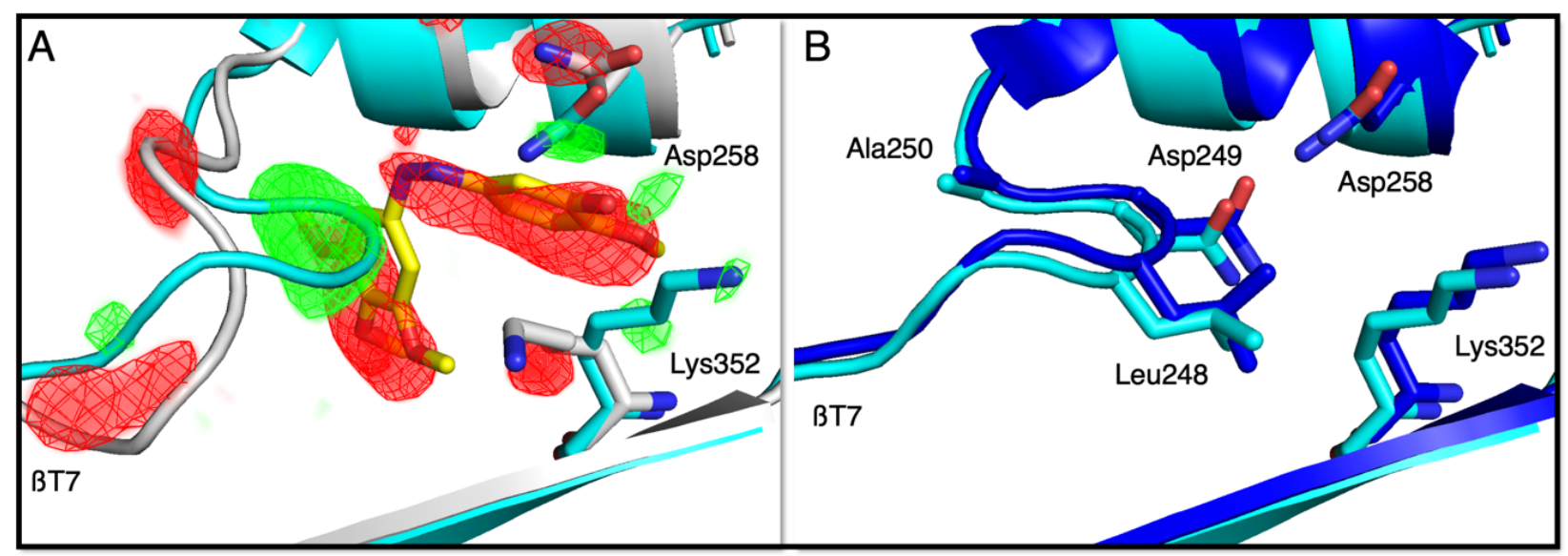

Fig. S4: Comparison of tubulin-azo-CA4 complex structures after photoinduced ligand release with a crystal structure obtained in the absence of any ligand. (A) Overlay of liganded dark (grey) and $100 \mathrm{~ms}$ (cyan) tubulin structures obtained by serial synchrotron crystallography. The corresponding difference densities (positive in green, negative in red, $\mathrm{F}_{\text {obs }}(100 \mathrm{~ms})-\mathrm{F}_{\text {obs }}(\mathrm{dark})$, sigma level $=5.0$ ) confirm the release of azo-CA4 and reorganization of the colchicine site in tubulin. (B) Overlay of the structure determined using the $100 \mathrm{~ms}$ data (cyan) and the one determined using data collected from unliganded crystals (blue). They are near identical and show that the release of azo-CA4 has been completed within this time regime. 


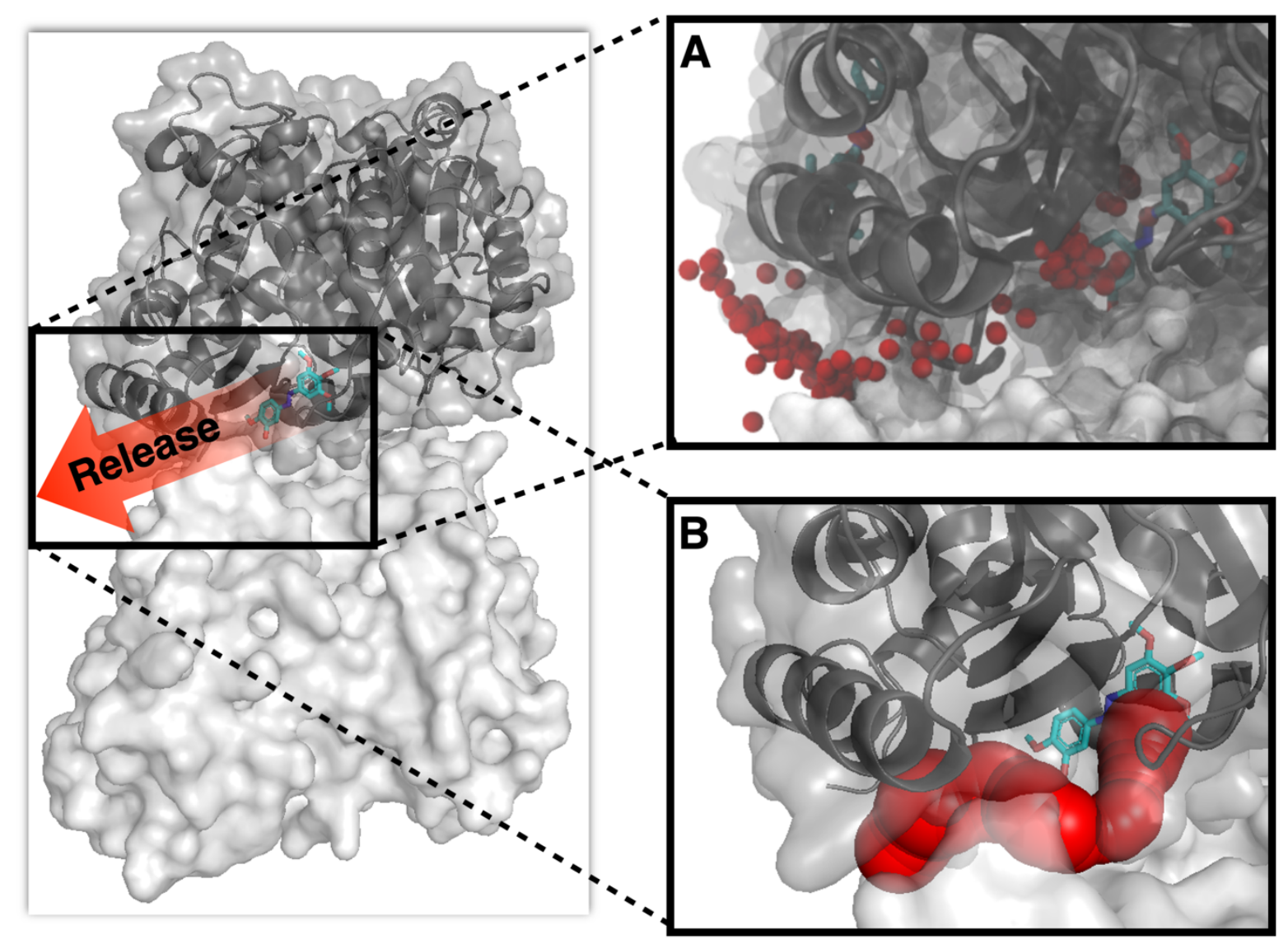

Fig. S5: Release pathway $1 \mathrm{~ms}$ after light illumination. (A) Unbinding pathway of azo-CA4 as obtained by enhanced sampling MD simulation. To obtain an unbinding event, Adiabatic-bias Molecular Dynamics (ABMD) simulations have been conducted using the electrostatic interactions between ligand and binding site residue heavy-atoms as collective variable (its target value was set to zero; elABMD (70)). The small red spheres depict the center-of-mass of ligand atoms, plotted every 50 frames of the simulation. They indicate a possible pathway azo-CA4 can take during the unbinding process. (B) The program Caver allows plotting of a release channel along the same pathway (Caver 3.0.3. azo-CA4 as specified starting point, minimum probe radius $=1.9 \AA$, shell depth $=4 \AA$, shell radius $=5 \AA$ ). 

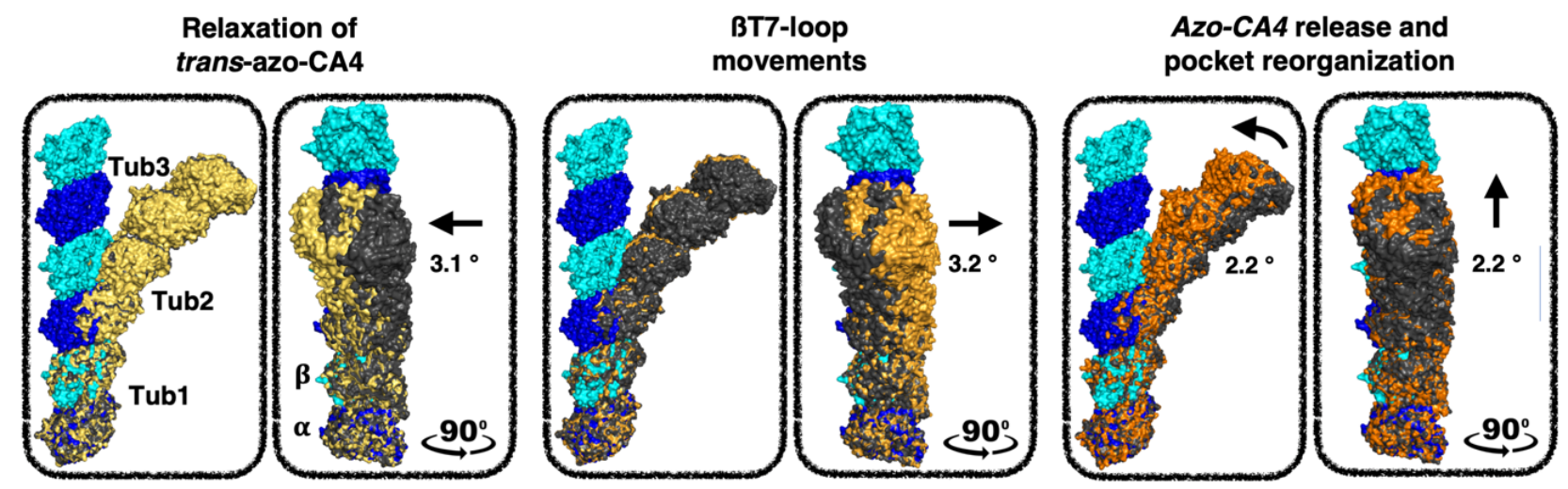

Fig. S6: Effects of protein backbone movements on the conformation of tubulin oligomers. Shown are three longitudinally aligned $\alpha \beta$-tubulin heterodimers as found in "straight" (PDB ID 5SYE (39); and "curved" microtubule protofilaments. The curved oligomers were generated by superimposing the structures of three consecutive $\alpha \beta$-tubulin heterodimers using their respective $\alpha$-tubulin subunit as a reference (33). The effects on oligomer conformation between different time delays are indicated by the angular changes occurring at the tip of each oligomer; they are defined as the differences between the centers of masses of the respective third tubulin dimers relative to the first dark tubulin dimer using PyMOL (Python script by Sean Law, Michigan State University). Angular deviations demonstrate the plasticity of tubulin during azo-CA4 relaxation in its binding pocket (125 ps in grey, $100 \mathrm{~ns}$ in yellow) and $\beta$ T7-loop movements of $\beta$-tubulin (100 ns in grey, $1 \mathrm{~ms}$ in bright orange), as well as a directed curvature adjustment towards the straight tubulin conformation after azo-CA4 release (1 ms in grey, $100 \mathrm{~ms}$ in orange). 
bioRxiv preprint doi: https://doi.org/10.1101/2022.02.17.480857; this version posted February 18,2022 . The copyright holder for this preprint (which was not certified by peer review) is the author/funder, who has granted bioRxiv a license to display the preprint in perpetuity. It is made available under aCC-BY-NC-ND 4.0 International license.

\begin{tabular}{|c|c|c|c|c|c|c|c|c|c|c|c|c|c|c|c|c|}
\hline & $\begin{array}{l}\text { Dark } \\
\text { (XFEL) }\end{array}$ & $\begin{array}{c}1 \mathrm{ps} \\
\text { (XFEL) }\end{array}$ & $\begin{array}{l}25 \mathrm{ps} \\
\text { (XFEL) }\end{array}$ & $\begin{array}{l}35 \mathrm{ps} \\
\text { (XFEL) }\end{array}$ & $\begin{array}{l}125 \mathrm{ps} \\
\text { (XFEL) }\end{array}$ & $\begin{array}{l}1 \mathrm{~ns} \\
\text { (XFEL) }\end{array}$ & $\begin{array}{l}10 \mathrm{~ns} \\
\text { (XFEL) }\end{array}$ & $\begin{array}{l}100 \text { ns } \\
\text { (XFEL) }\end{array}$ & $\begin{array}{c}1 \mu \mathrm{s} \\
\text { (XFEL) }\end{array}$ & $\begin{array}{l}10 \mu \mathrm{s} \\
\text { (XFEL) }\end{array}$ & $\begin{array}{l}100 \mu \mathrm{s} \\
\text { (XFEL) }\end{array}$ & $\begin{array}{c}1 \mathrm{~ms} \\
\text { (XFEL) }\end{array}$ & $\begin{array}{l}10 \mathrm{~ms} \\
\text { (XFEL) }\end{array}$ & $\begin{array}{l}\text { Apo } \\
\text { (XFEL) }\end{array}$ & $\begin{array}{l}\sim 100 \mathrm{~ms} \\
\text { (SYN) }\end{array}$ & $\begin{array}{l}\text { Dark } \\
\text { (SYN) }\end{array}$ \\
\hline \multicolumn{17}{|c|}{ Data collection } \\
\hline Space group & \multicolumn{16}{|c|}{$\mathrm{P} 2{ }_{1}$} \\
\hline$a, b, c(\AA \AA)$ & \multicolumn{13}{|c|}{$74.53,92.58,83.99$} & $\begin{array}{l}74.71 \\
92.71 \\
84.09\end{array}$ & \multicolumn{2}{|c|}{$74.28,91.87,83.69$} \\
\hline$\alpha, \beta, \gamma\left({ }^{\circ}\right)$ & \multicolumn{13}{|c|}{$90,96.71,90$} & $\begin{array}{c}90 \\
96.4 \\
90\end{array}$ & \multicolumn{2}{|c|}{$90,96.82,90$} \\
\hline Indexed patterns & 370356 & 55065 & 152814 & 62447 & 63962 & 63970 & 69058 & 61091 & 73798 & 67496 & 57915 & 60644 & 89399 & 82666 & 41314 & 102238 \\
\hline Indexing rate (\%) & 16.6 & 10.76 & 15.8 & 19.1 & 23.6 & 23.3 & 18.1 & 19.8 & 13.2 & 23.5 & 31.2 & 16.7 & 22.6 & 25.6 & 18.1 & 16.5 \\
\hline \multicolumn{14}{|c|}{$\begin{array}{l}\text { Overall statistics: } 11.09 \AA-1.70 \AA \\
\text { (high-resolution statistics: } 1.76 \AA-1.70 \AA \text { ) }\end{array}$} & & \multicolumn{2}{|c|}{$\begin{array}{l}\text { 92.11-2.1 } \\
(2.18-2.10 \AA)\end{array}$} \\
\hline No. reflections & $\begin{array}{l}123960 \\
(12356)\end{array}$ & $\begin{array}{l}122163 \\
(12154)\end{array}$ & $\begin{array}{l}122167 \\
(12154)\end{array}$ & $\begin{array}{l}122152 \\
(12154)\end{array}$ & $\begin{array}{c}122157( \\
12154)\end{array}$ & $\begin{array}{c}122155( \\
12154)\end{array}$ & $\begin{array}{l}122150 \\
(12154)\end{array}$ & $\begin{array}{l}122150 \\
(12154)\end{array}$ & $\begin{array}{l}122150 \\
(12154)\end{array}$ & $\begin{array}{l}122154 \\
(12154)\end{array}$ & $\begin{array}{l}122150 \\
(12154)\end{array}$ & $\begin{array}{l}122153 \\
(12154)\end{array}$ & $\begin{array}{l}122152 \\
(12154)\end{array}$ & $\begin{array}{l}104994 \\
(10494)\end{array}$ & $\begin{array}{l}38945 \\
(117)\end{array}$ & $\begin{array}{c}43965 \\
(945)\end{array}$ \\
\hline Completeness (\%) & $\begin{array}{c}100 \\
(100)\end{array}$ & $\begin{array}{c}100 \\
(100)\end{array}$ & $\begin{array}{c}100 \\
(100)\end{array}$ & $\begin{array}{c}100 \\
(100)\end{array}$ & $\begin{array}{c}100 \\
(100)\end{array}$ & $\begin{array}{c}100 \\
(100)\end{array}$ & $\begin{array}{c}100 \\
(100)\end{array}$ & $\begin{array}{c}100 \\
(100)\end{array}$ & $\begin{array}{c}100 \\
(100)\end{array}$ & $\begin{array}{c}100 \\
(100)\end{array}$ & $\begin{array}{c}100 \\
(100)\end{array}$ & $\begin{array}{c}100 \\
(100)\end{array}$ & $\begin{array}{c}100 \\
(100)\end{array}$ & $\begin{array}{c}100 \\
(100)\end{array}$ & $\begin{array}{l}60 \\
\text { (2) }\end{array}$ & $\begin{array}{c}67 \\
(15)\end{array}$ \\
\hline Multiplicity & $\begin{array}{c}2089.8 \\
(1000.6)\end{array}$ & $\begin{array}{c}268.3 \\
(107.6)\end{array}$ & $\begin{array}{c}893.1 \\
(447.5)\end{array}$ & $\begin{array}{c}378.2 \\
(200.9)\end{array}$ & $\begin{array}{c}416.8 \\
(210.6)\end{array}$ & $\begin{array}{c}365.9 \\
(188.4)\end{array}$ & $\begin{array}{c}347.5 \\
(175.9)\end{array}$ & $\begin{array}{c}314.1 \\
(164.8)\end{array}$ & $\begin{array}{c}404.2 \\
(208.4)\end{array}$ & $\begin{array}{c}392.6 \\
(207.5)\end{array}$ & $\begin{array}{c}309.1 \\
(155.8)\end{array}$ & $\begin{array}{c}328.9 \\
(161.2)\end{array}$ & $\begin{array}{c}586.1 \\
(313.1)\end{array}$ & $\begin{array}{c}429.5 \\
(194.3)\end{array}$ & $\begin{array}{c}274.2 \\
(216.6)\end{array}$ & $\begin{array}{c}602.5 \\
(504.6)\end{array}$ \\
\hline $\mathbf{R}_{\text {split }}(\%)$ & $\begin{array}{c}7.5 \\
(78.85)\end{array}$ & $\begin{array}{c}18.2 \\
(217.9)\end{array}$ & $\begin{array}{c}11.3 \\
(140.8)\end{array}$ & $\begin{array}{c}17.4 \\
(166.8)\end{array}$ & $\begin{array}{c}15.7 \\
(139.8)\end{array}$ & $\begin{array}{c}16.9 \\
(217.8)\end{array}$ & $\begin{array}{c}17.2 \\
(204.3)\end{array}$ & $\begin{array}{c}18.1 \\
(180.4)\end{array}$ & $\begin{array}{c}16.6 \\
(268.7)\end{array}$ & $\begin{array}{c}16.7 \\
(174.4)\end{array}$ & $\begin{array}{c}17.5 \\
(299.0)\end{array}$ & $\begin{array}{c}18.1 \\
(253.1)\end{array}$ & $\begin{array}{c}13.8 \\
(106.5)\end{array}$ & $\begin{array}{c}14.2 \\
(372.3)\end{array}$ & $\begin{array}{c}10.5 \\
(69.48)\end{array}$ & $\begin{array}{c}8.0 \\
(49.9)\end{array}$ \\
\hline$C C_{1 / 2}$ & $\begin{array}{c}0.992 \\
(0.694)\end{array}$ & $\begin{array}{c}0.965 \\
(0.221)\end{array}$ & $\begin{array}{c}0.987 \\
(0.449)\end{array}$ & $\begin{array}{c}0.967 \\
(0.354)\end{array}$ & $\begin{array}{c}0.972 \\
(0.431)\end{array}$ & $\begin{array}{c}0.971 \\
(0.265)\end{array}$ & $\begin{array}{c}0.968 \\
(0.274)\end{array}$ & $\begin{array}{c}0.964 \\
(0.323)\end{array}$ & $\begin{array}{c}0.972 \\
(0.210)\end{array}$ & $\begin{array}{c}0.971 \\
(0.343)\end{array}$ & $\begin{array}{c}0.969 \\
(0.170)\end{array}$ & $\begin{array}{c}0.968 \\
(0.217)\end{array}$ & $\begin{array}{c}0.979 \\
(0.558)\end{array}$ & $\begin{array}{l}0.981 \\
(0.14)\end{array}$ & $\begin{array}{c}0.992 \\
(0.528)\end{array}$ & $\begin{array}{c}0.996 \\
(0.660)\end{array}$ \\
\hline$\langle 1 / \sigma(I)\rangle$ & $\begin{array}{c}9.53 \\
(1.43)\end{array}$ & $\begin{array}{c}4.02 \\
(0.53)\end{array}$ & $\begin{array}{c}6.04 \\
(0.83)\end{array}$ & $\begin{array}{c}4.14 \\
(0.66)\end{array}$ & $\begin{array}{c}4.66 \\
(0.81)\end{array}$ & $\begin{array}{c}4.01 \\
(0.50)\end{array}$ & $\begin{array}{c}4.16 \\
(0.56)\end{array}$ & $\begin{array}{c}4.05 \\
(0.62)\end{array}$ & $\begin{array}{c}4.05 \\
(0.43)\end{array}$ & $\begin{array}{c}4.26 \\
(0.64)\end{array}$ & $\begin{array}{c}3.68 \\
(0.38)\end{array}$ & $\begin{array}{c}3.79 \\
(0.45)\end{array}$ & $\begin{array}{c}5.36 \\
(1.03)\end{array}$ & $\begin{array}{c}4.59 \\
(0.32)\end{array}$ & $\begin{array}{c}7.87 \\
(1.25)\end{array}$ & $\begin{array}{l}10.09 \\
(1.62)\end{array}$ \\
\hline PDB Code & & & & & & & & & & & & & & & & \\
\hline
\end{tabular}

\section{Table S1: Data Statistics}


bioRxiv preprint doi: https://doi.org/10.1101/2022.02.17.480857; this version posted February 18, 2022. The copyright holder for this preprint (which was not certified by peer review) is the author/funder, who has granted bioRxiv a license to display the preprint in perpetuity. It is made available under aCC-BY-NC-ND 4.0 International license.

\begin{tabular}{|c|c|c|c|c|c|c|c|c|c|c|c|c|c|c|c|c|}
\hline & $\begin{array}{l}\text { Dark } \\
\text { (XFEL) }\end{array}$ & $\begin{array}{c}1 \mathrm{ps} \\
\text { (XFEL) }\end{array}$ & $\begin{array}{l}25 \mathrm{ps} \\
\text { (XFEL) }\end{array}$ & $\begin{array}{l}35 \mathrm{ps} \\
\text { (XFEL) }\end{array}$ & $\begin{array}{l}125 \mathrm{ps} \\
\text { (XFEL) }\end{array}$ & $\begin{array}{c}1 \mathrm{~ns} \\
\text { (XFEL) }\end{array}$ & $\begin{array}{l}10 \mathrm{~ns} \\
\text { (XFEL) }\end{array}$ & $\begin{array}{l}100 \mathrm{~ns} \\
\text { (XFEL) }\end{array}$ & $\begin{array}{c}1 \mu \mathrm{s} \\
\text { (XFEL) }\end{array}$ & $\begin{array}{l}10 \mu \mathrm{s} \\
\text { (XFEL) }\end{array}$ & $\begin{array}{l}100 \mu \mathrm{s} \\
\text { (XFEL) }\end{array}$ & $\begin{array}{c}1 \mathrm{~ms} \\
\text { (XFEL) }\end{array}$ & $\begin{array}{l}10 \mathrm{~ms} \\
\text { (XFEL) }\end{array}$ & $\begin{array}{c}\text { Apo } \\
\text { (XFEL) }\end{array}$ & $\begin{array}{c}\sim 100 \mathrm{~ms} \\
\text { (SYN) }\end{array}$ & $\begin{array}{l}\text { Dark } \\
\text { (SYN) }\end{array}$ \\
\hline \multicolumn{17}{|c|}{ Refinement } \\
\hline Resolution (Å) & $\begin{array}{c}9.5 \AA \AA- \\
1.7 \AA\end{array}$ & $\begin{array}{c}9.5 \AA- \\
2.2 \AA\end{array}$ & $\begin{array}{c}9.5 \AA- \\
2.2 \AA\end{array}$ & $\begin{array}{c}9.5 \AA \AA- \\
2.2 \AA\end{array}$ & $\begin{array}{c}9.5 \AA- \\
2.2 \AA\end{array}$ & $\begin{array}{c}9.5 \AA- \\
2.2 \AA\end{array}$ & $\begin{array}{c}9.5 \AA- \\
2.2 \AA\end{array}$ & $\begin{array}{c}9.5 \AA \AA- \\
2.2 \AA\end{array}$ & $\begin{array}{c}9.5 \AA \AA- \\
2.2 \AA\end{array}$ & $\begin{array}{c}9.5 \AA- \\
2.2 \AA\end{array}$ & $\begin{array}{c}9.5 \AA \AA- \\
2.2 \AA\end{array}$ & $\begin{array}{c}9.5 \AA- \\
2.2 \AA\end{array}$ & $\begin{array}{c}9.5 \AA- \\
2.2 \AA\end{array}$ & $\begin{array}{l}9.5 \AA- \\
1.75 \AA\end{array}$ & $\begin{array}{c}73.75 \AA- \\
2.1 \AA\end{array}$ & $\begin{array}{c}73.75 \AA- \\
2.1 \AA\end{array}$ \\
\hline No. reflections & $\begin{array}{c}123591 \\
(8816)\end{array}$ & $\begin{array}{l}54292 \\
(3737)\end{array}$ & $\begin{array}{l}54329 \\
(3692)\end{array}$ & $\begin{array}{l}53497 \\
(3866)\end{array}$ & $\begin{array}{l}53355 \\
(3831)\end{array}$ & $\begin{array}{l}53409 \\
(3901)\end{array}$ & $\begin{array}{l}53221 \\
(3910)\end{array}$ & $\begin{array}{l}52461 \\
(3763)\end{array}$ & $\begin{array}{l}52234 \\
(3710)\end{array}$ & $\begin{array}{l}53180 \\
(3840)\end{array}$ & $\begin{array}{l}52580 \\
(3778)\end{array}$ & $\begin{array}{l}53111 \\
(3915)\end{array}$ & $\begin{array}{l}53134 \\
(3657)\end{array}$ & $\begin{array}{c}102745 \\
(1827)\end{array}$ & $\begin{array}{c}38921 \\
(68)\end{array}$ & $\begin{array}{c}43943 \\
(652)\end{array}$ \\
\hline $\boldsymbol{R}_{\text {work }} / \boldsymbol{R}_{\text {free in } \%}$ & $\begin{array}{c}12.13 / \\
15.38\end{array}$ & $\begin{array}{c}34.03 / \\
38.39\end{array}$ & $\begin{array}{c}24.66 / \\
29.13\end{array}$ & $\begin{array}{c}30.73 / \\
34.86\end{array}$ & $\begin{array}{c}29.83 / \\
33.22\end{array}$ & $\begin{array}{c}31.11 / \\
35.19\end{array}$ & $\begin{array}{c}31.13 / \\
34.33\end{array}$ & $\begin{array}{c}31.10 / \\
35.53\end{array}$ & $\begin{array}{c}30.46 / \\
34.88\end{array}$ & $\begin{array}{c}29.87 / \\
35.48\end{array}$ & $\begin{array}{c}30.48 / \\
36.29\end{array}$ & $\begin{array}{c}30.18 / \\
35.17\end{array}$ & $\begin{array}{c}28.52 / \\
33.36\end{array}$ & $\begin{array}{c}17.51 / \\
21.42\end{array}$ & $\begin{array}{c}17.28 / \\
23.08\end{array}$ & $\begin{array}{c}17.76 / \\
22.64\end{array}$ \\
\hline No. atoms & 8482 & 8349 & 8436 & 8327 & 8239 & 8284 & 8352 & 8211 & 8217 & 8228 & 8262 & 8274 & 8210 & 8366 & 8198 & 8646 \\
\hline Protein & 8392 & 7965 & 7963 & 7925 & 7925 & 7915 & 7945 & 7915 & 7909 & 7907 & 7912 & 7935 & 7931 & 7990 & 7986 & 8392 \\
\hline Ligands & 90 & 57 & 57 & 57 & 57 & 57 & 57 & 57 & 57 & 57 & 57 & 57 & 33 & 34 & 34 & 90 \\
\hline Water & 544 & 299 & 388 & 317 & 229 & 284 & 322 & 211 & 223 & 236 & 265 & 254 & 218 & 314 & 150 & 164 \\
\hline$B$-factors & 44.82 & 22.96 & 33.82 & 34.84 & 39.70 & 34.34 & 33.25 & 34.64 & 40.97 & 35.09 & 35.52 & 30.69 & 41.84 & 49.64 & 39.94 & 42.16 \\
\hline Protein & 44.27 & 23.05 & 33.79 & 34.95 & 39.77 & 34.43 & 33.34 & 34.69 & 41.10 & 35.17 & 35.60 & 30.77 & 41.89 & 49.60 & 39.08 & 42.30 \\
\hline Ligands & 36.79 & 17.95 & 24.75 & 28.28 & 33.37 & 26.95 & 32.38 & 29.40 & 32.34 & 28.44 & 31.26 & 24.09 & 28.41 & 33.19 & 21.95 & 36.13 \\
\hline Water & 54.51 & 21.83 & 35.77 & 33.57 & 38.92 & 33.47 & 27.06 & 34.37 & 38.62 & 33.88 & 34.30 & 29.50 & 42.22 & 53.15 & 35.76 & 38.15 \\
\hline Bond lengths ( $(\AA)$ & 0.008 & 0.002 & 0.002 & 0.002 & 0.001 & 0.001 & 0.001 & 0.002 & 0.002 & 0.001 & 0.001 & 0.004 & 0.001 & 0.008 & 0.001 & 0.002 \\
\hline Bond angles $\left({ }^{\circ}\right)$ & 0.885 & 0.420 & 0.426 & 0.444 & 0.405 & 0.414 & 0.424 & 0.455 & 0.436 & 0.409 & 0.426 & 0.673 & 0.422 & 1.050 & 0.417 & 0.469 \\
\hline $\begin{array}{l}\text { Ramachandran fa- } \\
\text { vored / allowed / } \\
\text { outliers in } \%\end{array}$ & $\begin{array}{c}98.71 / \\
1.29 / \\
0.00\end{array}$ & $\begin{array}{c}96.63 / \\
3.17 / \\
0.20\end{array}$ & $\begin{array}{c}96.83 / \\
2.97 / \\
0.20\end{array}$ & $\begin{array}{c}97.33 / \\
2.48 / \\
0.20\end{array}$ & $\begin{array}{c}97.33 / \\
2.48 / \\
0.20\end{array}$ & $\begin{array}{c}96.73 / \\
3.17 / \\
0.10\end{array}$ & $\begin{array}{c}97.23 / \\
2.57 / \\
0.20\end{array}$ & $\begin{array}{c}96.93 / \\
2.77 / \\
0.30\end{array}$ & $\begin{array}{c}96.73 / \\
2.87 / \\
0.40\end{array}$ & $\begin{array}{c}97.23 / \\
2.57 / \\
0.20\end{array}$ & $\begin{array}{c}96.82 / \\
2.68 / \\
0.50\end{array}$ & $\begin{array}{c}96.04 / \\
3.47 \\
0.50\end{array}$ & $\begin{array}{c}96.13 / \\
3.57 \\
0.30\end{array}$ & $\begin{array}{c}97.54 / \\
2.36 / \\
0.10\end{array}$ & $\begin{array}{c}95.97 / \\
3.54 / \\
0.49\end{array}$ & $\begin{array}{c}97.72 / \\
2.08 / \\
0.20\end{array}$ \\
\hline PDB Code & & & & & & & & & & & & & & & & \\
\hline
\end{tabular}

Table S2: Refinement Statistics 
Movie S1: Evolution of difference electron density along the azo-CA4 ligand over time. The movie shows the overlay of dark (grey) and time-resolved (protein in blue, azoCA4 in orange) structures with plotted electron difference density $\left(\mathrm{F}_{\text {obs }}(\right.$ light $)-\mathrm{F}_{\text {obs }}(\mathrm{dark})$, negative in red and positive in green). The contour level is shown at $3 \sigma$ for most time delays. For better comparison it is shown to higher levels for the 25 ps time delay where we collected about three times the light images and for the $\sim 100 \mathrm{~ms}$ time delay from the synchrotron experiment where we achieved higher activation levels.

Movie S2: Movie illustrating the principal steps upon azo-CA4 release. The movie is prepared from morphs between the thirteen molecular snapshots and highlights the effect of azo-CA4 (before and after illumination as yellow or orange sticks) isomerization, relaxation, release and the resulting collapse of the colchicine binding site ( $\alpha$-tubulin in blue and $\beta$-tubulin in cyan and $\beta$ T7 loop in green, respectively, cartoon or surface representation, selected residues as sticks). 